\title{
ASYMPTOTIC DEPENDENCE FOR LIGHT-TAILED HOMOTHETIC DENSITIES
}

\author{
GUUS BALKEMA, ${ }^{*}$ University of Amsterdam \\ NATALIA NOLDE, ${ }^{* *}$ University of British Columbia
}

\begin{abstract}
Dependence between coordinate extremes is a key factor in any multivariate risk assessment. Hence, it is of interest to know whether the components of a given multivariate random vector exhibit asymptotic independence or asymptotic dependence. In the latter case the structure of the asymptotic dependence has to be clarified. In the multivariate setting it is common to have an explicit form of the density rather than the distribution function. In this paper we therefore give criteria for asymptotic dependence in terms of the density. We consider distributions with light tails and restrict attention to continuous unimodal densities defined on the whole space or on an open convex cone. For simplicity, the density is assumed to be homothetic: all level sets have the same shape. Balkema and Nolde (2010) contains conditions on the shape which guarantee asymptotic independence. The situation for asymptotic dependence, treated in the present paper, is more delicate.
\end{abstract}

Keywords: Asymptotic dependence; homothetic level set; multivariate extreme; lighttailed density; geometric approach

2010 Mathematics Subject Classification: Primary 60G55; 60G70; 62E20

\section{Introduction}

In many real-world applications involving multivariate data, dependence between extreme observations in marginal components plays an important role in risk assessment and risk management. It is often the simultaneous occurrence of (unfavourable) extremes in several components that results in the failure of systems in the context of, for example, civil and reliability engineering, and leads to insolvency of financial institutions. In the latter context, the presence of joint extremes undermines benefits of risk diversification. Mathematically, we interpret asymptotic dependence in the context of max-stable limit laws; see, e.g. [3] for details. A max-stable limit law which is a product measure corresponds to asymptotic independence, and implies that large values in the marginal components of the data are unlikely to occur simultaneously.

The present paper gives a simple geometric condition on the density which guarantees asymptotic dependence. Assuming that the stochastic behaviour of the data may be described by a multivariate probability density, we exploit the geometric structure of the (asymptotic) shape of the level sets of the density in order to obtain information on dependence between coordinatewise extremes. This geometric approach recognizes the role that graphical visualization of the

Received 17 October 2011.

* Postal address: Department of Mathematics, University of Amsterdam, Science Park 904, 1098XH Amsterdam, The Netherlands. Email address: a.a.balkema@uva.nl

** Postal address: Department of Statistics, University of British Columbia, 6356 Agricultural Road, Vancouver, BC V6T 1Z2, Canada. Email address: natalia@stat.ubc.ca 
data plays in statistical analysis and gives insight into complicated analytical expressions. We restrict attention to densities with light tails. The case of heavy-tailed densities is less delicate and has been described in [2, Theorem 3.3]. For simplicity, we assume that level sets of the density are all scaled copies of some given set $D$. The set $D$ describes the common shape of the level sets of the density. Densities with this property are called homothetic. It is possible to relax this condition of homothetic densities slightly, as will be discussed in Section 5.

The asymptotic behaviour of the scaled random samples, or sample clouds, from light-tailed homothetic densities is determined by the shape of the level sets of the underlying density (cf. Proposition 4.2 of [2] and see also Figure 2 below). A well-known result, going back to [4], says that the components of a random vector with the multivariate normal density are asymptotically independent. The same holds for spherical and elliptical densities, but also for homothetic densities for which the common shape of the level sets is determined by a convex set with a smooth boundary, a set with a unique tangent plane at every boundary point (see Theorem 1.1 of [2]). As above, let $D \subset \mathbb{R}^{d}$ be the common shape of the level sets of a lighttailed homothetic density on $\mathbb{R}^{d}$. Assume that $D$ is a bounded open star-shaped set. Precise conditions are given below. Without loss of generality, let $\sup D=\boldsymbol{e}=(1, \ldots, 1)$. Here the sup is taken coordinatewise. The projection $D_{i}$ of $D$ on the $i$ th coordinate is an open interval with upper endpoint 1 . The assumption on the upper endpoints of the projections $D_{i}$ is a matter of convenience. It can be achieved by a proper scaling of the axes. The bivariate projection $D_{i j}$ is blunt if $(1,1)$ is not a boundary point of $D_{i j}$. The coordinates $X_{i}$ and $X_{j}$ of a vector $\boldsymbol{X}$ with a light-tailed homothetic density are asymptotically independent if $D_{i j}$ is blunt. See [2, Theorem 3.2]. From multivariate extreme value theory, it is known that, for light-tailed distributions, asymptotic independence implies that the exponent measure lives on the $d$ coordinate lines at minus infinity and has an exponential density on each of these lines. The corresponding Poisson point process on these lines describes the asymptotic behaviour of the sample clouds.

Here our focus is on asymptotic dependence. Since bluntness implies asymptotic independence, for asymptotic dependence, we want $\boldsymbol{e}=\sup D$ to be a boundary point of $D$. We want the set $D$ to be sharp. We shall impose two conditions on the translated set $D-\boldsymbol{e} \subset(-\infty, 0)^{d}$ : a local condition which says that $D-\boldsymbol{e}$ should be asymptotic to an open cone $C \subset(-\infty, 0)^{d}$ at the origin, and a global condition which says that $D-\boldsymbol{e}$ should be contained in a convex open cone whose closure, apart from the origin, lies in the open negative orthant. If the shape set $D$ is convex, these two cones may be chosen equal; for star-shaped sets, the local and the global conditions are different. Consider Figure 1. Asymptotic dependence holds for homothetic densities with exponential radial rate of decay and with level sets shaped like the triangle $T$ in Figure 1(a), but not for the shapes in Figure 1(b) and (c). For the shape in Figure 1(b), we can show that the high risk scenarios (see [1]) converge to a Gauss-exponential limit since the

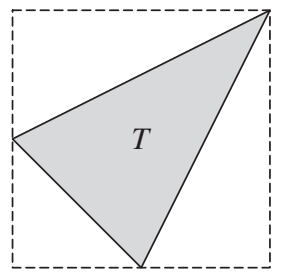

(a)

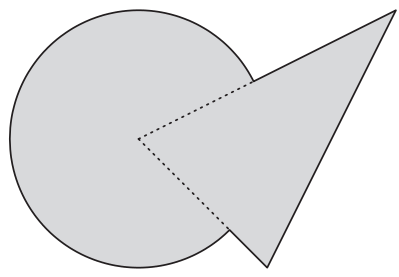

(b)

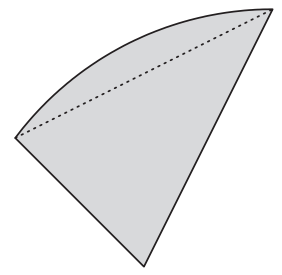

(c)

FIGURE 1: Examples of sets used in the introduction to discuss sharp sets and asymptotic dependence. 
contribution of the triangle becomes negligible compared to the contribution of the disk for horizontal half-planes high up. This example motivates the global condition above. The shape in Figure 1(c) generates a cone $C$ bounded by rays $(-t, 0), t>0$, and $(-t,-2 t)$. This implies asymptotic independence (by Theorem 4.2 below) even though the closure $\mathrm{cl} D$ intersects the lines $x=1$ and $y=1$ in one point only, the point $(1,1)$. The latter example explains the need of the rather stringent conditions in our definition of a sharp set.

If the shape set $D$ satisfies the two conditions above and if the density along the ray through the point $\boldsymbol{e}$ decreases at the right exponential rate asymptotically, then the coordinatewise maxima have a nondegenerate limit law. The exponent measure associated with the max-stable limit distribution has a density of a simple form. This density is the intensity of the Poisson point process which describes the asymptotic behaviour of the sample clouds in the direction of the diagonal. We shall now first describe a simple bivariate example.

\subsection{An example}

The example in this section is intended to illustrate the main ideas of the results to be developed in the later sections.

Consider a homothetic function $f$ on $\mathbb{R}^{2}$ with level sets $D_{t}=\left\{f>\mathrm{e}^{-t}\right\}=t D, t>0$. Suppose that $D$ is the triangle $T$ with vertices $(1,1),(-1,0)$, and $(0,-1)$; see Figure $1(\mathrm{a})$. In order to give an analytic expression for the function $f$, let $n_{T}$ denote the gauge function of the shape set $T ; n_{T}: \mathbb{R}^{2} \rightarrow[0, \infty)$ is a homogeneous function such that $T=\left\{n_{T}<1\right\}$. Then $f$ can be written as $f=\mathrm{e}^{-n_{T}}$. For the triangle above, we can write down $n_{T}$ explicitly as the maximum of three linear functions:

$$
n_{T}(x, y)=\max (-x-y, 2 x-y, 2 y-x), \quad(x, y) \in \mathbb{R}^{2} .
$$

Set $\boldsymbol{e}:=(1,1)$. Then the set $D_{t}=t T$ translated over $-t \boldsymbol{e}=(-t,-t)$ increases to the cone

$$
C=\left\{(x, y) \in(-\infty, 0)^{2} \mid 2 x<y<\frac{1}{2} x\right\},
$$

and $D_{s+t}-t \boldsymbol{e}$ converges to the shifted cone $C+s \boldsymbol{e}$ for $s \in \mathbb{R}$ as $t \rightarrow \infty$. Define a function $h$ on $\mathbb{R}^{2}$ with conic level sets:

$$
\left\{h>\mathrm{e}^{-t}\right\}=C+t \boldsymbol{e}, \quad t \in \mathbb{R} .
$$

Since $\boldsymbol{e}=(1,1)$ lies on the boundary of $T, f(t \boldsymbol{e})=\mathrm{e}^{-t}$, and we obtain the limit relation

$$
h_{t}(\boldsymbol{u})=\frac{f(t \boldsymbol{e}+\boldsymbol{u})}{f(t \boldsymbol{e})} \rightarrow h(\boldsymbol{u}) \quad \text { as } t \rightarrow \infty, \boldsymbol{u} \in \mathbb{R}^{2} .
$$

In fact, here $h_{t}$ and $h$ are equal outside a shifted quadrant, i.e.

$h_{t}(\boldsymbol{u})=\exp \left(-\max \left(2 u_{1}-u_{2}, 2 u_{2}-u_{1}\right)\right) \equiv h(\boldsymbol{u}), \quad \boldsymbol{u}=\left(u_{1}, u_{2}\right) \in \mathbb{R}^{2} \backslash(-\infty,-t)^{2}, t \geq 0$,

since $t T \equiv C+t \boldsymbol{e}$ on the complement of $(-\infty,-t)^{2}$.

The convergence in (1.1) is uniform on compact sets. Hence, the measures $\rho_{t}$ with density $h_{t}$ converge vaguely on the plane to the measure $\rho$ with density $h$. Note that $\rho H$ is finite for all half-planes $H=\{\xi \geq c\}$, with $\xi$ a linear functional and $c \in \mathbb{R}$, for which $\{\xi \geq-1\}$ intersects the cone $C$ in a bounded set, in particular for the horizontal half-planes $\{y \geq c\}$ and the vertical half-planes $\{x \geq c\}$. Observe that the function $h$ above satisfies the stability 
relation $h(\boldsymbol{u}+t \boldsymbol{e})=\mathrm{e}^{-t} h(\boldsymbol{u}), t \in \mathbb{R}$. This implies that the measure $\rho$ is an excess measure (see [1]) with the one-parameter group of symmetries:

$$
\rho(A+t \boldsymbol{e})=\mathrm{e}^{-t} \rho(A), \quad t \in \mathbb{R},
$$

for all Borel sets $A$ in the plane. Applied to the sets $A=\{x \geq t\}$ and $\{y \geq t\}$ this shows that the margins $\rho^{(1)}$ and $\rho^{(2)}$ of the measure $\rho$ satisfy the same symmetry relations:

$$
\rho^{(i)}[t, \infty)=\mathrm{e}^{-t} \rho^{(i)}[0, \infty), \quad i=1,2, t \in \mathbb{R} .
$$

This implies that $\rho^{(i)}$ is the exponential measure on $\mathbb{R}$ with density $c_{i} \mathrm{e}^{-t}$, where $c_{i}=\rho^{(i)}[0, \infty)$, $i=1,2$.

The above results for a general star-shaped set $D$ with a continuous boundary are formalized later in Theorem 3.1.

Observe that

$$
\int_{\mathbb{R}^{2}} \mathrm{e}^{-n_{T}(\boldsymbol{x})} \mathrm{d} \boldsymbol{x}=\int_{0}^{\infty} \mathrm{e}^{-s} \mathrm{~d}|s T|=2|T| \int_{0}^{\infty} s \mathrm{e}^{-s} \mathrm{~d} s=2|T|=3,
$$

where $|T|$ denotes the area of the triangle $T$. Hence, $f / 3$ is a probability density. Then so is $f(t \boldsymbol{e}+\boldsymbol{u}) / 3$. The factor $\frac{1}{3}$ drops out in the quotient in (1.1). Choose $t_{n}$ such that $f\left(t_{n} \boldsymbol{e}\right) / 3=1 / n$, i.e. $t_{n}=\log (n / 3)$. Then $\rho_{t_{n}}$, the measure with density $h_{t_{n}}$, has mass $n$, and if $\boldsymbol{X}_{1}, \boldsymbol{X}_{2}, \ldots$ are independent observations from the density $f / 3$, then $\rho_{t_{n}}$ is the mean measure of the sample cloud

$$
N_{n}=\left\{\boldsymbol{X}_{1}-t_{n} \boldsymbol{e}, \ldots, \boldsymbol{X}_{n}-t_{n} \boldsymbol{e}\right\}
$$

and these sample clouds converge in distribution to the Poisson point process $N$ with intensity $h$ vaguely on $\mathbb{R}^{d}$.

The monotone convergence $h_{t} \uparrow h$ implies $\mathcal{L}^{1}$-convergence on all half-planes on which $h$ is integrable, in particular on the horizontal and vertical half-planes. Hence, $\rho_{t} \rightarrow \rho$ weakly on these half-planes, and $N_{n} \Rightarrow N$ weakly. In particular, the coordinatewise maximum of the points in $N_{n}$ will converge in distribution to the coordinatewise maximum $\boldsymbol{W}$ of the points in $N$. In terms of distribution functions (DFs) we have

$$
G_{n}(\boldsymbol{u}):=F^{n}\left(t_{n} \boldsymbol{e}+\boldsymbol{u}\right) \rightarrow G(\boldsymbol{u}) \quad \text { weakly on } \mathbb{R}^{2},
$$

where $F$ denotes the DF with density $f / 3$. If we write $R(\boldsymbol{w})$ for the mass $\rho(E)$ of the complement $E$ of the shifted negative quadrant $(-\infty, \boldsymbol{w}]$ and $N(E)$ for the number of points of $N$ in the set $E$, then

$$
G(\boldsymbol{w})=\operatorname{Pr}\{\boldsymbol{W} \leq \boldsymbol{w}\}=\operatorname{Pr}\{N(E)=0\}=\mathrm{e}^{-\rho(E)}=\mathrm{e}^{-R(\boldsymbol{w})} .
$$

This tells us that $\rho$ is the exponent measure of the max-stable limit distribution $G$. The margins $G_{i}$ of $G$ are determined by the margins $\rho^{(i)}$, i.e.

$$
G_{i}(t)=\exp \left(-\rho^{(i)}(t, \infty)\right)=\exp \left(-c_{i} \mathrm{e}^{-t}\right)=\Lambda\left(t-\log c_{i}\right), \quad i=1,2,
$$

where $\Lambda$ is the standard Gumbel distribution. Integrating $h$ over half-planes $\left\{u_{i} \geq 0\right\}$ for $i=1,2$ gives the normalizing constants $c_{1}=c_{2}=2$.

Figure 2(b) illustrates a bivariate random sample of 10000 points drawn from the density $f=\mathrm{e}^{-n_{T}} / 3$. To do the simulation, first pick a point from the triangle $T$, uniformly, and then 


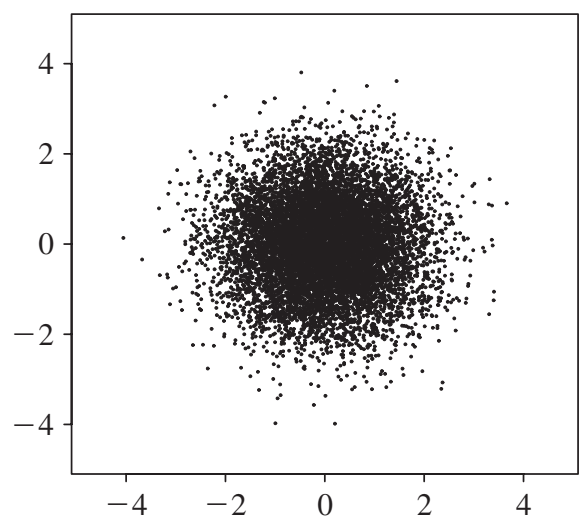

(a)

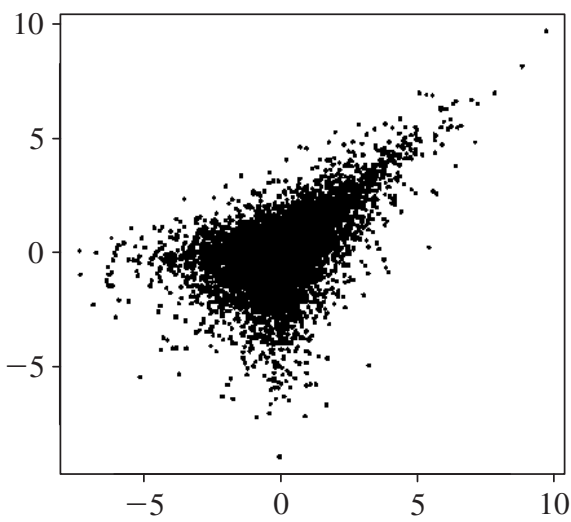

(b)

FIGURE 2: Simulated samples of 10000 points from the bivariate standard normal density (panel (a)) and the density $f=\mathrm{e}^{-n_{T}} / 3$ on $\mathbb{R}^{2}$ discussed in Section 1.1 (panel (b)).

simulate a positive random variable $R$ to determine the size of the triangle. Since the area increases quadratically, the variable $R$ has a gamma density $r^{2} \mathrm{e}^{-r} / 2, r>0$.

The components of the vector $\boldsymbol{X}$ with the density $\mathrm{e}^{-n_{T}} / 3$ above are asymptotically dependent. There is a max-stable limit vector $\boldsymbol{W}$ with dependent components since the exponent measure $\rho$ charges the plane $\mathbb{R}^{2}$. (It does not live on the two lines through $-\infty$.) The Poisson point process $N$ with mean measure $\rho$ gives a good impression of what the largest observations in a sample of size $n$ (for large values of $n$ ) from the density $\mathrm{e}^{-n_{T}} / 3$ will look like (after a translation over $\left.-t_{n} \boldsymbol{e}\right)$.

In this example we could use explicit computations for the constants, the trivial convergence $h_{t} \rightarrow h$ on bounded sets, and the monotone convergence $h_{t} \uparrow h$. Everything is easy if $D$ is a convex set which contains the origin and for which $\boldsymbol{q}=\sup D$ is a boundary point of $D$. The set $D-\boldsymbol{q}$ is asymptotic to an open convex cone $C, C=\cup n(D-\boldsymbol{q})$ and $C \supset D-\boldsymbol{q}$. If $\operatorname{cl}(C) \backslash\{\boldsymbol{0}\} \subset(-\infty, 0)^{d}$ then $D$ is sharp (the concept to be formally defined below), and $h=\mathrm{e}^{-n_{C}}$ is continuous and positive.

\subsection{The setting}

Asymptotic dependence is a more complex concept than asymptotic independence. In order to obtain results which do justice to this complexity without being overwhelmed by asymptotic conditions, the discussion of the present paper focuses on homothetic densities. We now introduce the definitions and notation which will be used in the sequel. More details can be found in [2, Section 3.1].

Consider an open bounded set $D \subset \mathbb{R}^{d}$, containing the origin. There exists a unique function, $n_{D}: \mathbb{R}^{d} \rightarrow[0, \infty)$, such that $D=\left\{\boldsymbol{x} \in \mathbb{R}^{d} \mid n_{D}(\boldsymbol{x})<1\right\}$ and $n_{D}(t \boldsymbol{x})=t n_{D}(\boldsymbol{x})$ for $t>0$ and $\boldsymbol{x} \in \mathbb{R}^{d}$. The function $n_{D}$ is called the gauge function of the set $D$. It is a norm with the open unit ball $D$ if $D$ is convex and if $-D=D$. It is continuous precisely if each ray intersects the boundary of $D$ in exactly one point; see [2, Proposition 3.2].

In order to incorporate homothetic densities which live on the open half-space $\left\{x_{d}>0\right\}$ or on the positive orthant, we introduce the following definition. 
Definition 1.1. A set $D$ is star shaped if $x \in D$ implies that $t \boldsymbol{x} \in D$ for all $0<t<1$. The class $D_{d}$ consists of all bounded open star-shaped sets $D \subset \mathbb{R}^{d}$ for which the cone $D_{\infty}=\bigcup_{n} n D$ is convex and for which the gauge function $n_{D}$ is continuous on $D_{\infty}$.

Homothetic densities on $\mathbb{R}^{d}$ generalize spherically symmetric densities. They have level sets which are scaled copies of a given bounded set $D \subset \mathbb{R}^{d}$ rather than the open unit ball $B$. These densities have a simple structure, and can be written down explicitly in the form $f(\boldsymbol{x})=$ $f_{0}\left(n_{D}(\boldsymbol{x})\right)$ for $\boldsymbol{x} \in \mathbb{R}^{d}$, where $n_{D}$ is the gauge function of the shape set $D$, and $f_{0}:[0, \infty) \rightarrow$ $(0, \infty)$ is the density generator. We take the set $D$ to be in the class $\mathcal{D}_{d}$. The density generator $f_{0}$ is assumed to be asymptotic to a von Mises function. Recall that von Mises functions have the asymptotics of exponential functions. A function $\mathrm{e}^{-\psi}$ on $[0, \infty)$ is a von Mises function with scale function $a(t)=1 / \psi^{\prime}(t)$ if

$$
\psi \text { is a } C^{2} \text { function with } \psi^{\prime}>0 \text {, and } a^{\prime}(t) \rightarrow 0 \text { for } t \rightarrow \infty \text {. }
$$

For a von Mises function $f_{0}$ with scale function $a$, the following limit relation holds (see, e.g. [3, p. 42]):

$$
f_{t}(u):=\frac{f_{0}(t+a(t) u)}{f_{0}(t)} \rightarrow \mathrm{e}^{-u} \quad \text { weakly on } \mathbb{R} \text { as } t \rightarrow \infty .
$$

This limit relation also holds in $\mathcal{L}^{1}$ on $[c, \infty)$ for all $c \in \mathbb{R}$.

\subsection{Outline of the paper}

In Section 2 we introduce the definitions and the notation related to the notions of sharp sets and cones, which will be used in the sequel. Section 3 contains the key technical results. Inspired by the example of the triangle in Section 1.1, we shall ask the following questions. Let $f=f_{0}\left(n_{D}\right)$ be a density as described in Section 1.2. What conditions on the shape of the set $D$ will ensure convergence of the functions $h_{t}$ in (1.1) and of the associated normalized sample clouds from density $f$ ? This is investigated in Section 3. Asymptotic dependence is described in Section 4. Finally, we discuss extensions outside the class of homothetic densities in Section 5 with some concluding remarks in Section 6 . Appendix A contains a number of counterexamples and supplementary results referred to in the main body of the paper.

\section{Preliminaries}

Definition 2.1. A nonempty open set $C$ is an open cone if $x \in C$ implies that $t x \in C$ for $t>0$. The cone is convex if $\boldsymbol{x}, \boldsymbol{y} \in C$ implies that $a \boldsymbol{x}+b \boldsymbol{y} \in C$ for $a, b>0$.

In [2] it was shown that, for a continuous positive light-tailed homothetic density, for asymptotic independence, it suffices that its level sets are blunt. In order to state conditions for asymptotic dependence, we need the notion of a sharp set, which we will introduce next.

Let us consider the bivariate situation first. Intuitively, the set $D \in \mathscr{D}_{2}$ is sharp if $\sup D=\boldsymbol{q}$ is a boundary point of $D$, and if there exist constants $a_{1}, a_{2}$ with $0<a_{1}<a_{2}<\infty$ such that the translate $D-\boldsymbol{q}$ is contained in the open cone $\left\{(x, y) \in(-\infty, 0)^{2} \mid a_{2} x<y<a_{1} x\right\}$. The condition on the values of the constants $a_{1}, a_{2}$ ensures that the enclosing cone is sharp. Its closure has no points with zero coordinates apart from the origin. In analogy with this bivariate case, we can define sharp cones and sharp sets in $\mathbb{R}^{d}$.

Definition 2.2. Suppose that $C$ is an open cone in $(-\infty, 0)^{d}$. Let

$$
C_{*}=C \cap\left\{x_{1}+\cdots+x_{d}=-1\right\} .
$$


The cone $C$ is sharp if the closure $\operatorname{cl}\left(C_{*}\right)$ is contained in the open simplex $(-\infty, 0)^{d} \cap\left\{x_{1}+\right.$ $\left.\cdots+x_{d}=-1\right\}$. Let $D \in \mathscr{D}_{d}$. The set $D$ is sharp if $\sup D=\boldsymbol{q}$ is a boundary point of $D$, and if the translate $D-\boldsymbol{q}$ is contained in a sharp open cone in $(-\infty, 0)^{d}$.

Example 2.1. For the cube $D=(-1,1)^{3}$, the bivariate projections $D_{i j}$ are neither blunt nor sharp. This remains true if we remove the part $\{x+y+z \geq 1\}$ containing the vertex $(1,1,1)$.

For an open cone in $(-\infty, 0)^{d}$ to be sharp, it suffices to check that some of its bivariate projections on coordinate planes are sharp. If $d$ is even then we need only check $d / 2$ projections, for example, $C_{12}, C_{34}, \ldots, C_{(d-1) d}$.

Proposition 2.1. Let $C$ be an open cone in $(-\infty, 0)^{d}$. If the bivariate projections $C_{i_{k} j_{k}}$ are sharp, and if the two-point sets $\left\{i_{k}, j_{k}\right\}$ cover $\{1, \ldots, d\}$, then $C$ is also sharp.

Proof. All points in the closure of $C$ have nonzero coordinates with the exception of the origin.

Sharpness alone does not suffice for asymptotic dependence. Even if $D \in \mathcal{D}_{2}$ is sharp, the uniform density on $D, c_{1} \mathbf{1}_{D}$, may be asymptotically independent, and so too the densities $c f_{0}\left(n_{D}\right)$ for von Mises functions $f_{0}$, as will be shown in Example A.4 in Appendix A. In addition to the global property of sharpness, we need good local behaviour. We shall assume that $D$ behaves asymptotically like a cone in the boundary point $\boldsymbol{q}$.

Definition 2.3. Let $O$ be a bounded open set in $\mathbb{R}^{d}, \boldsymbol{q}$ be a boundary point of $O$, and $C$ be an open cone in $\mathbb{R}^{d}$. The set $O$ is asymptotic to the cone $C$ in the point $\boldsymbol{q}$ if the translate $O_{0}=O-q$ has the following properties.

1. If $z_{n} \rightarrow z \in C$ and $r_{n}>0$ tends to 0 , then $r_{n} z_{n} \in O_{0}$ eventually.

2. If $z_{n} \rightarrow z \in(\mathrm{cl} C)^{c}$ and $r_{n}>0$ tends to 0 , then $r_{n} z_{n} \in\left(\mathrm{cl} O_{0}\right)^{c}$ eventually.

Example 2.2. Suppose that $O$ is a bounded convex set and that the origin is a boundary point. Then $r O \subset t O$ for $0<r<t$, and $O$ is asymptotic to the convex cone $C=\cup n O$ in the origin.

If $D \in \mathscr{D}_{d}$ is convex and asymptotic to $C$ in $\boldsymbol{q} \in \partial D$, then $C$ is convex. If $D-\boldsymbol{q} \subset\{\xi<0\}$ for a linear functional $\xi$ then $(C+\boldsymbol{q}) \cap\{\xi>0\}$ is a bounded convex set. This remains true if we replace 'convex' by 'star shaped'; see Proposition A.2 in Section A.2.2. However, $(C+\boldsymbol{q}) \cap\{\xi>0\}$ need not have a continuous boundary; see Example A.2.

Definition 2.4. The open cone $C$ belongs to the class $\mathcal{C}(\boldsymbol{q})$ for a nonzero vector $\boldsymbol{q}$ if $-\boldsymbol{q} \in \operatorname{cl}(C)$ and if there exists a linear functional $\xi$ such that the intersection $(\boldsymbol{C}+\boldsymbol{q}) \cap\{\xi=0\}$ lies in $D_{d-1}$ (it is a nonempty open subset of the hyperplane $\{\xi=0\}$, bounded, star shaped, with a continuous boundary, and the cone generated by it is convex).

Remark 2.1. Definition 2.4 does not depend on the choice of the linear functional $\xi$. Let $C^{0}$ be the interior of the convex cone of all linear functionals $\xi$ which are negative on $C$. Any $\xi \in C^{0}$ has the property that $C$ intersects the hyperplane $\{\xi=-\xi \boldsymbol{q}\}$ in a bounded set $C^{\xi}$. The sets $C^{\xi}$ for different $\xi$ are related by a central projection, and, hence, are homeomorphic. If one of these sets, translated to $\{\xi=0\}$, is star shaped with a continuous boundary then all are. Since the projection maps intervals into intervals, the cone generated by $C^{\xi}-\boldsymbol{q}$ is convex for all $\xi \in C^{0}$ if this holds for one such $\xi$.

Remark 2.2. It is possible that $C+\mathbb{R} \boldsymbol{q}$ is a proper cone even when $D_{\infty}=\mathbb{R}^{d}$, even for $d=2$. 
The nonnegative function $h$ with level sets $\left\{h>\mathrm{e}^{-t}\right\}=C+t \boldsymbol{q}$ is continuous and positive on $C+\mathbb{R} \boldsymbol{q}$, and zero on the complement of this open set. The condition $C \in \mathcal{C}(\boldsymbol{q})$ ensures that the intersection of $C+\boldsymbol{q}$ and the hyperplane $\{\xi=0\}$ for $\xi \in C^{0}$ generates a convex open cone in this hyperplane, and, hence, $C+\mathbb{R} \boldsymbol{q}$ is a convex open cone. If $-\boldsymbol{q} \in C$ then $C+\mathbb{R} \boldsymbol{q}=\mathbb{R}^{d}$, and $h$ is continuous and positive on $\mathbb{R}^{d}$.

\section{Convergence}

\subsection{Almost everywhere convergence}

Let $f=f_{0}\left(n_{D}\right)$ be a light-tailed homothetic density as described in Section 1.2, where $f_{0}$ is asymptotic to a von Mises function on $[0, \infty)$ with scale function $a(t)$ and $D \in \mathcal{D}_{d}$. Assume that $D$ is asymptotic to the cone $C \in \mathcal{C}(\boldsymbol{q})$ in a boundary point $\boldsymbol{q}$ of $D$. Let $h \geq 0$ have level sets $\left\{h>\mathrm{e}^{-t}\right\}=C+t \boldsymbol{q}, t \in \mathbb{R}$. Define

$$
h_{t}(\boldsymbol{u}):=\frac{f(t \boldsymbol{q}+a(t) \boldsymbol{u})}{f(t \boldsymbol{q})}, \quad \boldsymbol{u} \in \mathbb{R}^{d} .
$$

The almost everywhere (a.e.) convergence $h_{t} \rightarrow h$ follows from the convergence of the level sets, as will be shown below. If $-\boldsymbol{q}$ lies in the cone $C$ then the translates $C+n \boldsymbol{q}$ cover the whole space, and $h$ is continuous and positive on $\mathbb{R}^{d}$. The convergence $h_{t} \rightarrow h$ then holds uniformly on compact sets. If $-\boldsymbol{q}$ is a boundary point of $C$, the situation is less simple. The function $h$ is now continuous and positive on $C+\mathbb{R} \boldsymbol{q}$, and vanishes outside the closure of this convex cone. Its behaviour on the boundary is discussed in Appendix A. The convergence $h_{t} \rightarrow h$ holds uniformly on compact sets which do not intersect the boundary of the cone $C+\mathbb{R} \boldsymbol{q}$.

Proposition 3.1. Let $h \geq 0$ have level sets $\left\{h>\mathrm{e}^{-t}\right\}=C+t \boldsymbol{q}$ with $-\boldsymbol{q}$ a nonzero vector in the closure of the open cone $C$. If $C$ lies in $\mathcal{C}(\boldsymbol{q})$ then $h$ is continuous on $C+\mathbb{R} \boldsymbol{q}$.

Proof. Let $C \in \mathcal{C}(\boldsymbol{q})$. Write $z=(\boldsymbol{x}, y) \in \mathbb{R}^{d}$. Assume that $\boldsymbol{q}=\boldsymbol{e}_{d}$ is the vertical unit vector in $\mathbb{R}^{d}$ and $C+\boldsymbol{q}$ intersects $\{y=0\}$ in a set $D \in \mathcal{D}_{d-1}$. The gauge function $n_{D}$ is continuous on $D_{\infty}$ by Definition 1.1, and, hence, so is $\tilde{n}_{D}(\boldsymbol{x}, y)=n_{D}(\boldsymbol{x})+y$. Conclude that $h=\mathrm{e}^{-\tilde{n}_{D}}$ since the level sets of $\tilde{n}_{D}$ are translates of $\left\{\tilde{n}_{D}<0\right\}=\left\{(\boldsymbol{x}, y) \mid y<-n_{D}(\boldsymbol{x})\right\}$.

The level sets of $h_{t}$ in (3.1) are affine copies of $D$. They have the form

$$
\left\{h_{t}>c\right\}=r_{t}(D-\boldsymbol{q})+v_{t} \boldsymbol{q} .
$$

Set $a_{t}=a(t)$. For $c=1$, by construction, $v_{t}=0$ and $r_{t}=t / a_{t}$; for general $c>0$,

$$
r_{t}=\frac{t}{a_{t}}+v_{t}, \quad f_{0}\left(t+v_{t} a_{t}\right)=c f_{0}(t) .
$$

The quotient $t / a_{t}$ diverges since $a_{t} \ll t$ for scale functions, and $v_{t} \rightarrow-\log c$. By assumption, $D$ is asymptotic to the open cone $C$ in $\boldsymbol{q} \in \partial D$. Hence, the bounded open set in (3.2) is an approximation to $\{h>c\}=C-(\log c) \boldsymbol{q}$.

Proposition 3.2. If $z_{n} \rightarrow z \in\{h>0\}$ and $t_{n} \rightarrow \infty$, then $h_{t_{n}}\left(z_{n}\right) \rightarrow h(z)$. If $z$ is an interior point of $\{h=0\}$ then $h_{t_{n}}\left(z_{n}\right) \rightarrow 0$.

Proof. First assume that $-\boldsymbol{q} \in C$. Then $h$ is continuous and positive on the whole space. Let $z_{n} \rightarrow z$. If $h(z)>\mathrm{e}^{-s}$ then $z-s \boldsymbol{q} \in C$ and $z_{n}-s \boldsymbol{q} \in r_{n}(D-\boldsymbol{q})$ eventually for any sequence $r_{n} \rightarrow \infty$ by statement 1 of Definition 2.3. Hence, for $s_{0}>s$ eventually, $h_{t_{n}}\left(z_{n}\right)>\mathrm{e}^{-s_{0}}$. 
Similarly, $h(z)<\mathrm{e}^{-s}$ implies that $z-s \boldsymbol{q}$ lies outside the closure of $C$ by continuity of $h$, and, hence, by statement 2 of Definition $2.3, z_{n}-s \boldsymbol{q}$ eventually lies outside the closure of $r_{n}(D-\boldsymbol{q})$, and $h_{t_{n}}\left(z_{n}\right)<c$ for any $c>\mathrm{e}^{-s}$.

Now assume that $C+\mathbb{R} \boldsymbol{q}$ is a proper cone. If $z$ lies in this cone, the argument above remains valid, and $h_{t_{n}}\left(z_{n}\right) \rightarrow h(z)$ whenever $z_{n} \rightarrow z \in C+\mathbb{R} \boldsymbol{q}$ and $t_{n} \rightarrow \infty$. So let $z_{n} \rightarrow \boldsymbol{z}$, where $z$ lies in the interior of the complement of this cone. Let $s>1$. There is an open cone $C^{*}$ which contains $\operatorname{cl}(C) \backslash\{\boldsymbol{0}\}$ such that $z-s \boldsymbol{q} \notin \operatorname{cl}\left(C^{*}\right)$. This then also holds for $z_{n}$ for $n \geq n_{0}$. Let $C^{*} \subset\{\xi<0\}$ for some linear functional $\xi$, and let $z \in\{\xi>-M\}$ for some $M>1$. This then also holds for $z_{n}$ for $n \geq n_{1}$. For $r>r_{0}$,

$$
r(D-\boldsymbol{q}) \subset C^{*} \quad \text { on }\{\xi>-M\}-s \boldsymbol{q} .
$$

Hence, for $r>r_{0}$, the points $z$ and $z_{n}, n \geq n_{0} \vee n_{1}$, do not lie in $r(D-\boldsymbol{q})+s \boldsymbol{q}$, which implies that $h_{t}\left(z_{n}\right) \leq \mathrm{e}^{-s / 2}$ for $t \geq t_{0}$.

\subsection{An inequality}

The $\mathcal{L}^{1}$-convergence $h_{t} \rightarrow h$ on a half-space $H$ follows if we have appropriate bounds on the level sets $H \cap\left\{h_{t}>\mathrm{e}^{-n}\right\}, n \geq 0$, which are uniform in $t \geq t_{0}$. Sharpness of $D$ implies that $D-\boldsymbol{q}$ is contained in a proper open convex cone $Q$. The level sets in (3.2) are then contained in translates of $Q$. We shall derive a bound on the size of the intersections $H \cap\left(Q+t_{n} \boldsymbol{q}\right)$. The vector $\boldsymbol{q}$ is nonzero and $\boldsymbol{- q}$ lies in the closure of $Q$. This ensures that the sequence $Q_{n}=Q+s_{n} \boldsymbol{q}$ is increasing if we assume that

$$
s_{n}=S_{1}+\cdots+S_{n}, \quad 0<S_{n+1}<\mathrm{e}^{\varepsilon} S_{n}, \quad n \geq 1 .
$$

Our bound depends on $\varepsilon>0$. The bound allows us to show that the integral of $\min \left(h_{t}, \mathrm{e}^{-m}\right)$ is small uniformly in $t \geq t_{0}$. For simplicity, we may assume below that $\xi=y$, the vertical coordinate. Then $Q$ lies in the lower half-space $\{y \leq 0\}$.

Proposition 3.3. Let $\varepsilon \in(0,1 / 2 d]$, where $d \geq 1$ is the dimension of our space. Let $C$ be a convex open cone, and let $\boldsymbol{q}$ be a nonzero vector such that $-\boldsymbol{q} \in \operatorname{cl}(C)$. Define a sequence of cones $C_{n}=C+s_{n} \boldsymbol{q}$, where the $s_{n}$ satisfy (3.3). Then $s_{m} / s_{1} \leq m \mathrm{e}^{m / 2 d}$ for $m \geq 1$. Let $g \geq 0$ be a measurable function on $\mathbb{R}^{d}$ whose level sets satisfy $\left\{g>\mathrm{e}^{-n}\right\} \subset C_{n}$ for $n \geq 0$. Let $H:=\{\xi \geq-M\}$ intersect $C$ in a bounded nonempty set. Then $c_{0}:=\xi \boldsymbol{q}$ is positive and the volume $b_{1}:=|\{\xi \geq-1\} \cap C|$ is finite. There exists an integer $m_{d}$ such that

$$
J_{m}:=\int_{H \backslash C_{m}} g(z) \mathrm{d} z \leq c \mathrm{e}^{-m / 3}, \quad m \geq m_{d},
$$

where $c=\mathrm{e} b_{1}\left(c_{0} s_{1}+M\right)^{d}$, and $m_{d}$ depends only on the dimension $d$.

Proof. Since $\left\{g>\mathrm{e}^{-n}\right\} \subset C_{n}, n \geq 0$, we have $g \leq \mathrm{e}^{1-n}$ on the complement of $C_{n-1}$ and, hence, also on $C_{n} \backslash C_{n-1}$ and on $\left(C_{n} \backslash C_{n-1}\right) \cap H$. This gives (see Figure 3)

$$
J_{m} \leq \mathrm{e} \sum_{n>m} \mathrm{e}^{-n}\left|C_{n} \cap H\right|=\mathrm{e} \sum_{n>m} \mathrm{e}^{-n}\left(c_{0} s_{n}+M\right)^{d} b_{1} .
$$

From the condition on the points $s_{n}$, we obtain the crude bounds

$$
s_{n} \leq\left(1+\mathrm{e}^{\varepsilon}+\mathrm{e}^{2 \varepsilon}+\cdots+\mathrm{e}^{(n-1) \varepsilon}\right) s_{1} \leq n \mathrm{e}^{n \varepsilon} s_{1} \quad \text { and } \quad c_{0} s_{n}+M \leq\left(c_{0} s_{1}+M\right) n \mathrm{e}^{n \varepsilon},
$$




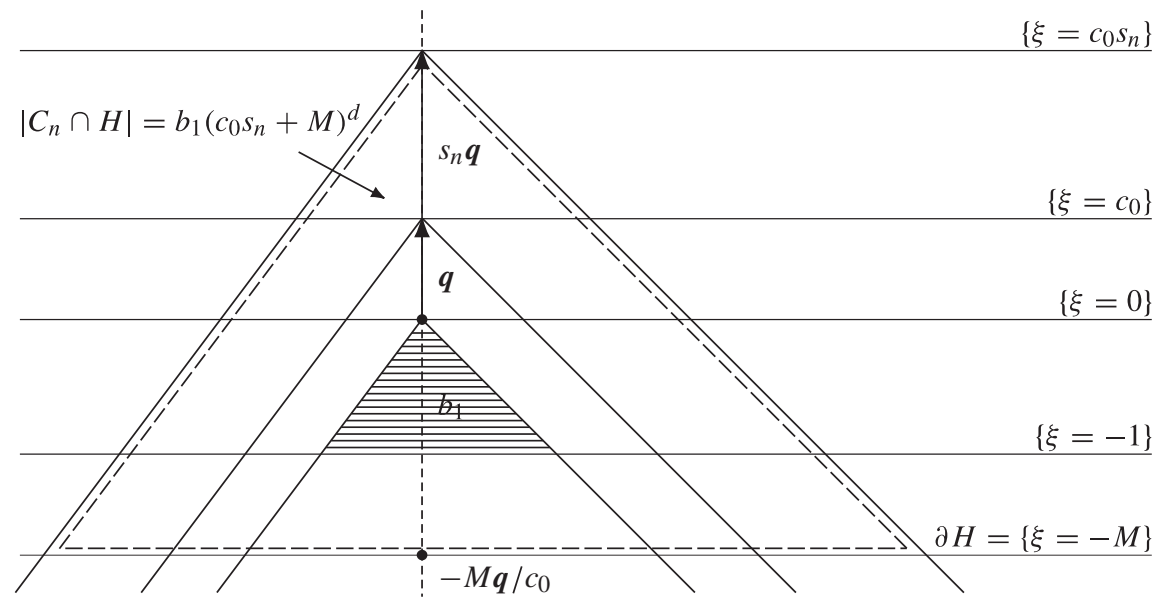

FIgURe 3: An illustrative plot used in the proof of Proposition 3.3.

from which we obtain the bound for $s_{m} / s_{1}$ in the proposition, and shows that $J_{m} \leq \mathrm{e} b_{1}\left(c_{0} s_{1}+\right.$ $M)^{d} \sum_{n>m} \mathrm{e}^{-n} n^{d} \mathrm{e}^{n \varepsilon d}$. Since $\varepsilon d \leq \frac{1}{2}$ by assumption, the series in the previous expression can be bounded by $\sum_{n>m} n^{d} \mathrm{e}^{-n / 2}$, which is bounded by $\mathrm{e}^{-m / 3}$ for $m \geq m_{d}$ as may be seen by comparing the sum with an integral.

Given the open convex cone $C$, a nonzero vector $-\boldsymbol{q}$ in the closure of $C$ and a half-space $H$ intersecting $C$ in a bounded nonempty set, and $\varepsilon \in(0,1 / 2 d]$ for any $\delta>0$, we can find a bounded set $C_{m} \cap H$ such that the integral $J_{m}$ over $H \backslash C_{m}$ is bounded by $\delta$. This bound holds uniformly for all nonnegative measurable functions $g$ whose level sets satisfy the inclusions $\left\{g>\mathrm{e}^{-n}\right\} \subset C_{n}$ for $n \geq 0$. Actually, the inclusion need only hold for $n \geq m$. If a sequence of such functions $g$ converges a.e. on $H$ and is uniformly bounded on $H$, then it converges in $\mathcal{L}^{1}$ on $H$. This follows by uniform integrability; see Lemma A.1 in Appendix A.

\section{3. $\mathscr{L}^{1}$-convergence}

For a boundary point $\boldsymbol{q}$ of a bounded open star-shaped set $D$, there are two open cones associated with $D$. The cone $C$ describes the asymptotic behaviour of the set $D-\boldsymbol{q}$ at the origin. It may not exist in every boundary point. The larger cone $Q$ encloses $D-\boldsymbol{q}$. For $Q$, we take the interior of the closed convex cone which is the intersection of all half-spaces $\{\xi \geq 0\}$ which contain $D$. Large sample clouds from the homothetic density $f_{0}\left(n_{D}\right)$ will have the shape of $D$ if $f_{0}$ decreases rapidly. We are interested in the trace of such sample clouds on half-spaces $H=\{\xi \geq c\}$ which cut off a tip of this sample cloud around the extreme point on the ray through $\boldsymbol{q}$, but which contain no other points. The proper condition here is that $\{\xi \geq-1\}$ intersects $Q$ in a bounded nonempty set. This condition implies that the origin is the only boundary point of $D-\boldsymbol{q}$ in the hyperplane $\{\xi=0\}$.

Theorem 3.1. Let $D \in D_{d}, \boldsymbol{q} \in \partial D$, and $C \in \mathcal{C}(\boldsymbol{q})$. Assume that $D$ is asymptotic to the cone $C$ in $\boldsymbol{q}$, and let $Q$ denote the interior of the intersection of all closed half-spaces $\{\xi \geq 0\}$ which contain $D-\boldsymbol{q}$. Let $h \geq 0$ be the function with level sets

$$
\left\{h>\mathrm{e}^{-t}\right\}=C+t \boldsymbol{q}, \quad t \in \mathbb{R} .
$$


Let $f=f_{0}\left(n_{D}\right)$ be a density where $f_{0}$ is a von Mises function with scale function a $(t)$. Then

$$
h_{t}(\boldsymbol{u})=\frac{f(t \boldsymbol{q}+a(t) \boldsymbol{u})}{f(t \boldsymbol{q})} \rightarrow h(\boldsymbol{u}) \text { a.e. on } \mathbb{R}^{d} .
$$

Let $\Gamma$ denote the set of nonzero linear functionals $\xi$ which satisfy

(*) $\{\xi=-1\}$ intersects $Q$ in a bounded set.

Then $\Gamma$ is a convex open cone, and $h_{t} \rightarrow h$ holds in $\mathcal{L}^{1}$ on all half-spaces $\{\xi \geq c\}$ with $\xi \in \Gamma$ and $c \in \mathbb{R}$.

Proof. The a.e. convergence $h_{t} \rightarrow h$ follows from Proposition 3.1 since the boundary of the convex cone $(C+\mathbb{R} \boldsymbol{q})$ is a null set.

The set $\Gamma$ is a convex open cone. It is a convex cone: if the half-spaces $\left\{\xi_{i} \geq-1\right\}$ intersect $Q$ in a bounded set then this holds for their union, and, hence, for all half-spaces $a \xi_{1}+b \xi_{2}$ with $a, b>0$. It is open: let $\boldsymbol{p} \neq \mathbf{0}$ and write $\xi \boldsymbol{x}=\boldsymbol{p}^{\top} \boldsymbol{x}$. The vector $\boldsymbol{p}$ is normal to the planes determined by $\xi$. Then $\xi$ satisfies $(*)$ precisely if $-\boldsymbol{p} \in Q$, and $Q$ is open.

$\mathcal{L}^{1}$-convergence now follows from the inequality in Proposition 3.3. First note that the graph of the function $h_{t}$ is obtained from that of $f$ by translating it over $-t \boldsymbol{q}$ then scaling it by $1 / a_{t}$ with $a_{t}=a(t)$ and, finally, multiplying the function by $1 / f_{0}(t)$. This means that $h_{t}$ has its maximum in the point $-r_{t} \boldsymbol{q}$, where $r_{t}=t / a_{t} \rightarrow \infty$. It assumes the value 1 in the origin, and the level set $\left\{h_{t}>1\right\}=r_{t}(D-\boldsymbol{q})$ supports the half-space $H_{0}=\{\xi \geq 0\}$ in $\mathbf{0}$ if $\xi \in \Gamma$. The function $h_{t}^{*}(s)=f_{0}\left(t+a_{t} s\right) / f_{0}(t)$ satisfies

$$
h_{t}^{*}(s) \rightarrow \mathrm{e}^{-s} \text { as } t \rightarrow \infty .
$$

If $D$ contains the origin then $h_{t}^{*}(s)=h_{t}(s \boldsymbol{q})$.

Let $\xi \in \Gamma$. For $M>0$, the half-space $H=\{\xi \geq-M\}$ intersects $Q$ in a bounded nonempty set. Then, for some multiple $N,-N \boldsymbol{q} \in \partial H$ and $N \xi \boldsymbol{q}=M$. Let $c_{t}:=h^{*}(-N)$. The level set $\left\{h_{t}>c_{t}\right\}$ supports $H$ in the point $-N \boldsymbol{q}$, i.e. $\left\{h_{t}>c_{t}\right\}$ is disjoint from $H$ but $-N \boldsymbol{q}$ is a common boundary point. This level set of $h_{t}$ has the form $r(D-\boldsymbol{q})-N \boldsymbol{q}$ for some $r>0$, and, hence, is contained in the shifted convex cone $Q-N \boldsymbol{q}$, which also supports $H$ in $-N \boldsymbol{q}$. Since $h_{t}^{*}(-N)<2 \mathrm{e}^{N}$ eventually, $h_{1}<2 \mathrm{e} N$ on $H$ for $t \geq t(H)$.

The function $h_{t}^{*}$ satisfies $h_{t}^{*}(0)=1$. It assumes the values $1 / \mathrm{e}^{n}$ in $s_{n}=S_{1}+\cdots+S_{n}$, where $0<S_{n+1}<\mathrm{e}^{\varepsilon} S_{n}$ for $t \geq t(\varepsilon)$; see Lemma 3.1 below. Moreover, $s_{1} \leq 2$ eventually by (3.5). Hence, we may apply the inequality with $C=Q$. Lemma A.1 then gives the $\mathcal{L}^{1}$-convergence $h_{t} \rightarrow h$ on $H$.

Lemma 3.1. Let $f_{0}$ be a continuous positive function on $[0, \infty)$ which is asymptotic to a von Mises function $\mathrm{e}^{-\psi}$ with scale function a; see (1.2). Given $t \geq 0$, choose $t<t^{\prime}<t^{\prime \prime}$ such that $f_{0}\left(t^{\prime}\right)=f_{0}(t) / \mathrm{e}$ and $f_{0}\left(t^{\prime \prime}\right)=f_{0}\left(t^{\prime}\right) / \mathrm{e}$. For any $\varepsilon>0$, there exists $t_{0}$ such that $t^{\prime \prime}-t^{\prime}<\mathrm{e}^{\varepsilon}\left(t^{\prime}-t\right)$ for $t \geq t_{0}$.

Proof. The function $f_{0}$ satisfies $f_{0}(t+x a(t)) / f_{0}(t) \rightarrow \mathrm{e}^{-x}$ weakly on $\mathbb{R}$ and, hence, uniformly on $[c, \infty)$ for any $c \in \mathbb{R}$. This implies that $t^{\prime}-t$ and $t^{\prime \prime}-t$ are asymptotic to $a(t)$ and $2 a(t)$, respectively.

\subsection{Consequences}

This section contains three corollaries of Theorem 3.1. For half-spaces $H_{t}=\{\xi \geq t\}$ with $\xi \in \Gamma$, there exists a simple asymptotic expression for $\operatorname{Pr}\left\{\boldsymbol{X} \in H_{t}\right\}$ for $t \rightarrow \infty$. 
The $\mathcal{L}^{1}$-convergence $h_{t} \rightarrow h$ implies convergence of the corresponding measures, and this implies convergence of the sample clouds to a Poisson point process $N$ with intensity $h$. The conditions of the theorem are quite general. If we go back to the original setting where sup $D=\boldsymbol{e}$ and $D$ was supposed to be sharp in $\boldsymbol{e}$, then we obtain results about the convergence of coordinatewise maxima and the max-stable limit distribution, or rather the exponent measure associated with this limit distribution.

Corollary 3.1. For $\xi \in \Gamma$, let $c(\xi)$ be the volume of $(C+\boldsymbol{q}) \cap H$, where $H=\{\xi \geq 0\}$. Then

$$
\operatorname{Pr}\{\boldsymbol{X} \in t \boldsymbol{q}+H\} \sim f_{0}(t) a(t)^{d} \Gamma(d+1) c(\xi) \text { as } t \rightarrow \infty .
$$

Proof. The $\mathcal{L}^{1}$-convergence of $h_{t} \rightarrow h$ on $H$ implies that

$$
\int_{H} h_{t}(\boldsymbol{u}) \mathrm{d} \boldsymbol{u} \rightarrow \int_{H} h(\boldsymbol{u}) \mathrm{d} \boldsymbol{u}=\int_{0}^{\infty} c(\xi) r^{d} \mathrm{de}^{-r}=\Gamma(d+1) c(\xi) .
$$

Hence,

$$
\int_{H+t \boldsymbol{q}} f(\boldsymbol{x}) \mathrm{d} \boldsymbol{x}=a(t)^{d} \int_{H} f(t \boldsymbol{q}+a(t) \boldsymbol{u}) \mathrm{d} \boldsymbol{u}=a(t)^{d} f_{0}(t) \int_{H} h_{t}(\boldsymbol{u}) \mathrm{d} \boldsymbol{u}
$$

gives the desired expression.

Corollary 3.2. Let $N$ be the Poisson point process on $\mathbb{R}^{d}$ with intensity $h$. This point process lives on the convex open cone $C+\mathbb{R} \boldsymbol{q}$. Set $a_{n}=a\left(t_{n}\right)$, where $t_{n}$ satisfies

$$
n a_{n}^{d} f_{0}\left(t_{n}\right) \rightarrow 1 \text { as } n \rightarrow \infty \text {. }
$$

Let $\boldsymbol{X}_{1}, \boldsymbol{X}_{2}, \ldots$ be independent observations from the density $f$. Then, for $\xi \in \Gamma$ and $c \in \mathbb{R}$,

$$
N_{n}:=\left\{\frac{\boldsymbol{X}_{1}-t_{n} \boldsymbol{q}}{a_{n}}, \ldots, \frac{\boldsymbol{X}_{n}-t_{n} \boldsymbol{q}}{a_{n}}\right\} \Rightarrow N \text { weakly on } H=\{\xi \geq c\} .
$$

Corollary 3.3. Suppose that $D_{i j}$ is sharp for $1 \leq i<j \leq d$. Then $\Gamma$ contains the d coordinate functions. The coordinatewise maxima converge:

$$
\boldsymbol{W}_{n}:=\max N_{n}=\frac{\left(\boldsymbol{X}_{1} \vee \cdots \vee \boldsymbol{X}_{n}\right)-t_{n} \boldsymbol{e}}{a_{n}} \Rightarrow \boldsymbol{W}:=\max N .
$$

The exponent measure of $\boldsymbol{W}$ lives on $\mathbb{R}^{d}$ and has density $h$. It does not charge the boundary at $-\infty$.

\section{Asymptotic dependence}

If the set $D \in \mathscr{D}_{d}$ in the boundary point $\boldsymbol{q}$ is asymptotic to a cone $C \in \mathcal{C}(\boldsymbol{q})$ then the sample clouds from a homothetic density $f=f_{0}\left(n_{D}\right)$ have a simple asymptotic description in the direction $\boldsymbol{q}$ provided the density generator $f_{0}$ is asymptotic to a von Mises function. This description sometimes makes it possible to decide whether coordinates are asymptotically independent.

Theorem 4.1. Let $D \in \mathscr{D}_{d}$ in the boundary point $\boldsymbol{q}$ be asymptotic to the cone $C \in \mathcal{C}(\boldsymbol{q})$. Let $N$ be the Poisson point process with mean measure $\rho$, where $\rho$ has density $h$ with level 
sets $\left\{h>\mathrm{e}^{-t}\right\}=C+t \boldsymbol{q}, t \in \mathbb{R}$. Let $\Gamma$ be the convex open cone of linear functionals determined by the open cone $Q$ in Theorem 3.1. Let $f_{0}$ be a von Mises function on $[0, \infty)$ with scale function $a(t)$. There exist positive numbers $t_{n} \uparrow \infty$ such that $n f_{0}\left(t_{n}\right) a\left(t_{n}\right)^{d} \rightarrow 1$. The function $f_{0}\left(n_{D}\right)$ is integrable. Let $\boldsymbol{X}_{1}, \boldsymbol{X}_{2}, \ldots$ be independent observations from the density $f=c f_{0}\left(n_{D}\right)$. The sample clouds converge weakly on all half-spaces $\{\xi \geq c\}, \xi \in \Gamma, c \in \mathbb{R}$ :

$$
N_{n}=\left\{\frac{\boldsymbol{X}_{1}-t_{n} \boldsymbol{q}}{a_{n}}, \ldots, \frac{\boldsymbol{X}_{n}-t_{n} \boldsymbol{q}}{a_{n}}\right\} \Rightarrow N, \quad a_{n}=a\left(t_{n}\right) .
$$

1. If $\Gamma$ contains the coordinate functions $x_{1}, \ldots, x_{m}$ then the coordinates $X_{1}, \ldots, X_{m}$ are asymptotically dependent. The exponent measure of the max-stable limit distribution lives on $\mathbb{R}^{m}$. It is the projection of the measure $\rho$.

2. If $\rho\left\{x_{1} \geq 0\right\}=\infty$ and $\Gamma$ contains a functional $x_{2}+$ ax $x_{1}$ for some $a \geq 0$, then $X_{1}$ and $\mathrm{X}_{2}$ are asymptotically independent.

Proof. Statement 1 follows from the continuous mapping theorem (see, e.g. [3, p. 152]) applied to the projection $\left(x_{1}, \ldots, x_{d}\right) \mapsto\left(x_{1}, \ldots, x_{m}\right)$. For statement 2 , first assume that $\rho\left\{x_{2} \geq 0\right\}=\infty$. Then $a$ is positive and $\rho\left\{x_{1}>0, x_{2}>0\right\}=c<\infty$. Let $\rho_{t}$ have density $h_{t}$ in (3.4), and let $\tilde{\rho}$ and $\tilde{\rho}_{t}$ be the projections of $\rho$ and $\rho_{t}$ on the $\left(x_{1}, x_{2}\right)$-plane. Let $Q=(0, \infty)^{2}$ be the positive quadrant. Then, for $i=1,2$,

$$
\operatorname{Pr}\left\{X_{1}>t q_{1}, X_{2}>t q_{2}\right\}=f_{0}(t) \tilde{\rho}_{t}(Q) \ll f_{0}(t) \tilde{\rho}_{t}\left\{x_{i}>0\right\}=\operatorname{Pr}\left\{X_{i}>t q_{i}\right\} \quad \text { as } t \rightarrow \infty .
$$

Asymptotic independence follows from Proposition A.1 with the continuous curve $t \mapsto\left(t q_{1}\right.$, $\left.t q_{2}\right)$. If $\tilde{\rho}\left\{x_{2}>0\right\}=c_{2}$ is finite then $c_{2}$ is positive since $\rho$ is a nonzero excess measure, $\tilde{\rho} Q=$ $c_{0} \leq c_{2}$, and $\tilde{\rho}_{t}\left(x_{1}>M, x_{2}>0\right\} \rightarrow \tilde{\rho}\left\{x_{1}>M, x_{2}>0\right\}=\mathrm{e}^{-M} c_{0}$. Hence, for any $\varepsilon>0$, there exists a continuous curve $\left(x_{1}(t), x_{2}(t)\right)$ such that $\operatorname{Pr}\left\{X_{1}>x_{1}(t), X_{2}>x_{2}(t)\right\}<\varepsilon p_{i}(t)$ eventually in the terminology of Proposition A.1. By the same proposition, this suffices for asymptotic independence.

Example 4.1. Let $C$ be a convex open cone whose closure lies in $(-\infty, 0)^{d} \cup\{\boldsymbol{0}\}$. Let $D \subset$ $(-1,1)^{d}$ be asymptotic to $C$ in the boundary point $\boldsymbol{e}=(1, \ldots, 1)$. Assume that $D$ has no other boundary points on any of the coordinate planes $\left\{x_{i}=1\right\}$. Let $f_{0}$ be a von Mises function. Then the function $f_{0}\left(n_{D}\right)$ is integrable, and the components of the vector $\boldsymbol{X}$ with density $f=c f_{0}\left(n_{D}\right)$ are asymptotically dependent. The exponent measure has density $h$ with level sets $\left\{h>\mathrm{e}^{-t}\right\}=C+t \boldsymbol{e}$ for $t \in \mathbb{R}$.

We can give exact conditions for asymptotic independence in the bivariate case if $D$ is convex. If $D$ is blunt, the coordinates are asymptotically independent as shown in [2]. So we may assume that $\sup D$ is a boundary point of $D$.

Theorem 4.2. Let $\left(X_{1}, X_{2}\right)$ have density $f_{0}\left(n_{D}\right)$, where $D$ is a bounded open convex set and $f_{0}$ is a von Mises function. Suppose that sup $D$ is a boundary point of $D$. Then $X_{1}$ and $X_{2}$ are asymptotically independent if and only if $D$ has a horizontal or vertical tangent at this point.

Proof. Convexity implies that $\Gamma$ consists of all linear functionals $\xi$ for which $\{\xi \geq-1\} \cap C$ is bounded. In particular, $u+v \in \Gamma$. If the tangent lines in $\boldsymbol{q}=\sup D$ are not horizontal or vertical then $D$ is sharp and $X_{1}$ and $X_{2}$ are asymptotically dependent. The exponent measure has density $h$ with level curves $C+\boldsymbol{q}$, where $C$ is the cone $a x<y<b x$ with $0<b<a<\infty$ the 
slopes of the tangent lines. If one of the tangent lines is horizontal or vertical, we may assume that there is a horizontal tangent by symmetry. Then $h(u, v)=\mathrm{e}^{-v}$ on $(-\infty, 0) \times(0, \infty)$, and, hence, $\rho\{v \geq 0\}=\infty$. On the other hand, $\rho(0, \infty)^{2}$ is finite. So statement 2 of Theorem 4.1 applies.

Example 4.2. Let $D$ be the intersection of the open disk of radius 2 centred in $(1,-1)$ and the open half-plane above the diagonal. The vector $(X, Y)$ with density $c \mathrm{e}^{-n_{D}}$ has asymptotically independent components. This is similar to the set depicted in Figure 1(c).

Example 4.3. If $D$ is the square $(-1,1)^{2}$ and $f_{0}$ is a von Mises function, then the vector $(X, Y)$ with density $f=c f_{0}\left(n_{D}\right)$ has asymptotically independent components.

Example 4.4. The cube $(-1,1)^{3}$ determines two open tetrahedra $D_{1}$ and $D_{2}$. Let $D_{1}$ have the vertices $(1,1,1),(1,-1,-1),(-1,1,-1)$, and $(-1,-1,1)$, and let $D_{2}$ have the other four vertices of the cube. The bivariate projections are squares. The two vectors with density $f=c_{0} \mathrm{e}^{-n_{D_{i}}}$ have asymptotically independent coordinates. The vertex $\boldsymbol{e}=(1,1,1)$ is a boundary point of $D_{1}$, but $D_{2}$ is disjoint from the unit ball centred in $\boldsymbol{e}$.

Example 4.5. Let $T$ be the open triangle with vertices $(1,1),(-1,0)$, and $(0,-1)$, and let $D$ be the intersection of $T \times(-1,1)$ with the half-spaces $\{z>x+y-2\}$ and $\{z<2-x-y\}$. The set $D$ is asymptotic to a cone $C$ in $(1,1,0)$. Let $(X, Y, Z)$ have density $c \mathrm{e}^{-n_{D}}$. Then $X$ and $Z$ are asymptotically independent, and so are $Y$ and $Z$, but $X$ and $Y$ are asymptotically dependent. There is a three-dimensional limit distribution for the coordinatewise maxima. The exponent measure lives on the union of the vertical line in $-\infty$ and the horizontal plane in $-\infty$. On the vertical line it has an exponential density; on the horizontal plane the density $h$ has level sets $\left\{h>\mathrm{e}^{-t}\right\}=C+(t, t)$, where $C$ is the cone $\{2 x<y<x / 2\}$.

\section{Extensions}

In this section we extend our results to certain light-tailed densities whose level sets are not homothetic.

Suppose that we have a continuous homothetic function $f=f_{0}\left(n_{D}\right)$ which vanishes off the convex cone $D_{\infty}=\cup n D$ for some $D \in \mathscr{D}_{d}$ and some continuous decreasing function $f_{0}$ asymptotic to a von Mises function $\mathrm{e}^{-\psi}$. The only difference with the standard setup in the previous pages is that we do not assume that $f$ integrates to 1 . We assume that there exists a continuous function $\tilde{f} \leq f$ which is a probability density. The level sets $\left\{\tilde{f}>\mathrm{e}^{-s}\right\}$ are then contained in the corresponding level sets $\left\{f>\mathrm{e}^{-s}\right\}$ of $f$. If the level sets of $\tilde{f}$ are asymptotic to those of $f$ and the coordinates of the vector with density $f$ are asymptotically dependent, this does not imply asymptotic dependence for the coordinates of the vector with density $\tilde{f}$.

Example 5.1. Let $f=\mathrm{e}^{-n_{T}}$ for the triangle $T$ in Figure 1(a), as in Section 1.1. Then $\{f>$ $\left.\mathrm{e}^{-s}\right\}=s T$ for $s>0$. Let $\tilde{f}$ have level sets $\tilde{T}_{s}=\left\{\tilde{f}>\mathrm{e}^{-s}\right\}$ which, for large $s$, are the triangle $s T$ from which a small triangle at the vertex $(s, s)$ has been removed. Suppose that $\tilde{T}_{s}=T_{s} \cap\{(x, y) \mid x+y<2 s-2 \sqrt{s}\}$. Then $\tilde{T}_{s} / s$ converges to $T$, but the translates $\tilde{T}_{s}-s \boldsymbol{e}$ do not converge to the cone $C$ but to the empty set. The random vector with a probability density which has the level sets $\tilde{T}_{s}$ for $s \geq s_{0}$ has components which are asymptotically independent; see [2, Example A.5].

Asymptotic equality of level sets does not preserve asymptotic dependence, but asymptotic equality of densities does. 
Proposition 5.1. Let $f$ and $\tilde{f}$ be continuous positive densities, and let $\tilde{f}\left(z_{n}\right) / f\left(z_{n}\right) \rightarrow 1$ for any sequence $z_{n}$ for which $\left\|z_{n}\right\| \rightarrow \infty$. Suppose that $f$ has bounded level sets $\left\{f>\mathrm{e}^{-s}\right\}$ for $s>0$. Then so has $\tilde{f}$. If $h_{t}:=f(a(t) \boldsymbol{w}+t \boldsymbol{q}) / f(t \boldsymbol{q}) \rightarrow h(\boldsymbol{w})$ a.e. and in $\mathcal{L}^{1}$ on $a$ half-space $H$, and $h$ is bounded on $H$, then $\tilde{h}_{t}:=\tilde{f}(a(t) \boldsymbol{w}+t \boldsymbol{q}) / \tilde{f}(t \boldsymbol{q}) \rightarrow h(\boldsymbol{w})$ a.e. and in $\mathcal{L}^{1}$ on $H$.

Proof. The conditions ensure that $\tilde{f}(t \boldsymbol{q}) \sim f(t \boldsymbol{q})$. The maximum of $f$ over the half-space $H_{t}:=a(t) H+t \boldsymbol{q}$ vanishes for $t \rightarrow \infty$. Hence, for any $r>1$ eventually, $H_{t} \cap r B$ is empty and, hence, $\tilde{f} / f \rightarrow 1$ uniformly on $H_{t}$. This implies that $\tilde{h}_{t} / h_{t} \rightarrow 1$ uniformly on $H$ for $t \rightarrow \infty$, which ensures the a.e. and $\mathcal{L}^{1}$-convergence of $\tilde{h}_{t}$.

The limit function $h$ has the property that $h(z+t \boldsymbol{q})=\mathrm{e}^{-t} h(z)$, reflecting the well-known symmetry of the exponent measure $\rho(A+t \boldsymbol{q})=\mathrm{e}^{-t} \rho(A)$ in the light-tailed case. It suffices to know $h$ on the horizontal coordinate plane $\{y=0\}$. Write $z=(\boldsymbol{x}, y)$. The restriction $h_{0}(\boldsymbol{x})=h(\boldsymbol{x}, 0)$ is a multivariate exponential function. It has the form

$$
h_{0}=\mathrm{e}^{-n_{E}}, \quad E=(C+\boldsymbol{q}) \cap\{y=0\} .
$$

The assumption that $C \in \mathcal{C}(\boldsymbol{q})$ ensures that $n_{E}$ is continuous on the convex open cone $E_{\infty}=$ $\cup n E$. Any function which is positive and continuous on a convex open cone and vanishes outside this cone, and which is exponential along each ray, with negative exponent depending on the direction of the ray, has this form provided that it vanishes at $\infty: h_{0}\left(\boldsymbol{x}_{n}\right) \rightarrow 0$ whenever $\left\|\boldsymbol{x}_{n}\right\| \rightarrow \infty$. This last condition ensures that the level sets are scaled copies of a set in $\mathscr{D}_{d-1}$. If the set $E$ is convex then $h_{0}$ is log-concave or strongly unimodal. The measure $\rho_{0}$ with density $h_{0}$, scaled by its integral, may be thought of as the spectral probability measure for the exponent measure $\rho$.

Instead of the horizontal coordinate plane we may use the restriction to one of the other coordinate planes to describe $h$. Actually, we may take any hyperplane $\{\xi=c\}$ in $\mathbb{R}^{d}$ as long as $\xi \boldsymbol{q} \neq 0$. If $\xi$ lies in the open cone $\Gamma$ in Theorem 3.1 then $h$ is integrable over the hyperplane. The condition that $\xi_{0} \in \Gamma$ is equivalent to the condition that the exponential function $h_{0}$ on the hyperplane $\left\{\xi_{0}=0\right\}$ is bounded away from 1 outside the unit ball. Thus, we have a simple correspondence: the exponent measure has a density $h$ with level sets $\left\{h>\mathrm{e}^{-t}\right\}=C+t \boldsymbol{q}$ and the spectral measure has a multivariate exponential density. The description of the restriction of $h$ to a hyperplane will depend on the hyperplane and on the coordinates used. The description of the cone $C=\{h>1\}$ is purely geometric and does not depend on coordinates.

Now let us return to the restriction $h_{0}$ of $h$ to the horizontal coordinate plane. Let $\tilde{h}_{0}$ be a continuous function such that $0 \leq \tilde{h}_{0} \leq h_{0}$. There is a unique extension to a continuous function $\tilde{h}$ on $\mathbb{R}^{d}$ which satisfies the symmetry relation $\tilde{h}(z+t \boldsymbol{q})=\mathrm{e}^{-t} \tilde{h}(z)$. Simply define $\tilde{h}((\boldsymbol{x}, 0)+t \boldsymbol{q})=\mathrm{e}^{-t} \tilde{h}_{0}(\boldsymbol{x})$. Let $\tilde{\rho}$ be the measure with density $\tilde{h}$, and let $\tilde{N}$ be the Poisson point process with intensity $\tilde{h}$. The intensity of the points of $\tilde{N}$ increases exponentially as one moves away in the direction of $-\boldsymbol{q}$, just as in the case of the original Poisson point process with intensity $h$. One may regard $\tilde{N}$ as a thinning of $N$. Each point $z$ of $N$ is retained with probability $\pi=\pi(z)$ depending on $z$, independent of what happens to the other points. Here $\pi(z)=\tilde{h}(z) / h(z)$. If the function $\tilde{h}_{0}$ vanishes on an open ball $P$ in $\{y=0\}$ then $\tilde{h}$ vanishes on the cylinder $P+\mathbb{R} \boldsymbol{q}$. All points of $N$ in this cylinder are deleted.

Now return to the situation above where $\tilde{f} \leq f$. If $f$ satisfies the conditions of Theorem 3.1, except for being a probability density, and if $\tilde{f}$ satisfies the limit relation below (with denominator $f_{0}(t)$ ), then this convergence holds in $\mathcal{L}^{1}$ on the same half-spaces as for $f$. 
Proposition 5.2. Suppose that $f=f_{0}\left(n_{D}\right)$ satisfies (3.4), where $f_{0}$ is a von Mises function on $[0, \infty)$ with scale function $a(t)$ and $D \in D_{d}$ is asymptotic to the cone $C \in \mathcal{C}(\boldsymbol{q})$ in the boundary point $\boldsymbol{q} \neq \mathbf{0}$ of $D$. Let $Q$ be the smallest convex cone which contains $D-\boldsymbol{q}$. Let $\tilde{f}$ be a density and $0 \leq \tilde{f} \leq f$, and assume that there exists a function $\tilde{h}$ continuous on the convex cone $C+\mathbb{R} \boldsymbol{q}$ and 0 on the complement of the cone such that

$$
\tilde{h}_{t_{n}}\left(\boldsymbol{w}_{n}\right)=\frac{\tilde{f}\left(a\left(t_{n}\right) \boldsymbol{w}_{n}+t_{n} \boldsymbol{q}\right)}{f_{0}\left(t_{n}\right)} \rightarrow \tilde{h}(\boldsymbol{w}) \text { as } t_{n} \rightarrow \infty, \boldsymbol{w}_{n} \rightarrow \boldsymbol{w} \in C+\mathbb{R} \boldsymbol{q} .
$$

Then $\tilde{h}(\boldsymbol{w}+t \boldsymbol{q})=\mathrm{e}^{-t} \tilde{h}(\boldsymbol{w})$ for $t \in \mathbb{R}, \boldsymbol{w} \in \mathbb{R}^{d}$, and the convergence $\tilde{h}_{t} \rightarrow \tilde{h}$ holds in $\mathcal{L}^{1}$ on all half-spaces $\{\xi \geq c\}$ with $\xi \boldsymbol{q}>0$ for which $\{\xi \geq-1\} \cap Q$ is bounded.

Proof. Set $t_{n}^{\prime}=t_{n}+s_{n} a\left(t_{n}\right)$. Let $\boldsymbol{w}_{n} \rightarrow \boldsymbol{w} \in C+\mathbb{R} \boldsymbol{q}$ and $s_{n} \rightarrow s \in \mathbb{R}$. Then $a\left(t_{n}^{\prime}\right) / a\left(t_{n}\right)=$ $c_{n} \rightarrow 1$ and

$$
\tilde{h}_{t_{n}^{\prime}}\left(\boldsymbol{w}_{n}\right)=\frac{\tilde{f}\left(t_{n} \boldsymbol{q}+s_{n} a\left(t_{n}\right) \boldsymbol{q}+a\left(t_{n}\right)\left(c_{n} \boldsymbol{w}_{n}\right)\right)}{f_{0}\left(t_{n}\right)} \frac{f_{0}\left(t_{n}\right)}{f_{0}\left(t_{n}^{\prime}\right)} \rightarrow \frac{\tilde{h}(\boldsymbol{w}+s \boldsymbol{q})}{\mathrm{e}^{-s}} .
$$

This proves the symmetry. The $\mathcal{L}^{1}$-convergence follows from a version of Lebesgue's dominated convergence theorem: if $f_{n}$ is measurable and $\left|f_{n}\right| \leq g_{n}$ for $n \geq 1$, and if $f_{n} \rightarrow f$ a.e. and $g_{n} \rightarrow g$ in $\mathcal{L}^{1}$, then $f_{n} \rightarrow f$ in $\mathcal{L}^{1}$.

\section{Concluding remarks}

In this paper we investigated the asymptotic dependence of the coordinates for vectors with light-tailed distributions. The distribution was assumed to be specified in terms of a multivariate density. For simplicity, we first developed the theory for continuous homothetic densities. The light-tailed homothetic densities of this paper were determined by a bounded open star-shaped set $D$ which describes the shape of the level sets, and a decreasing light-tailed function $f_{0}$ on $[0, \infty)$ which describes the rate of decrease. In analogy to spherically symmetric densities $f(\boldsymbol{x})=f_{0}(\|\boldsymbol{x}\|)$ we wrote $f(\boldsymbol{x})=f_{0}\left(n_{D}(\boldsymbol{x})\right)$ for homothetic densities. The densities considered in this paper are continuous and positive on the whole space or on a convex cone.

If $D$ is a convex set which contains the origin and whose boundary has a unique tangent plane in each point, then the coordinates of the random vector with density $f$ are asymptotically independent. In this paper we treated the case of asymptotic dependence and we assumed that the set $D$ has a vertex $\boldsymbol{q} \neq \mathbf{0}$. It is then often possible to give a simple description of extremes in the direction of $\boldsymbol{q}$. This description is in terms of a Poisson point process $N$ on $\mathbb{R}^{d}$. The asymptotic behaviour of the extremes is determined by the rate of decrease of $f_{0}$ and by two open cones, $C \subset Q$, associated with the set $D$. The cone $C$ describes the asymptotic behaviour of $D$ at the vertex $\boldsymbol{q}$; the cone $Q$ is the smallest convex open cone containing the translate $D-\boldsymbol{q}$. The intensity $h$ of the Poisson point process $N$ is determined by the cone $C$. The intensity is positive and continuous on the convex cone $C+\mathbb{R} \boldsymbol{q}$; its level sets are translates of $C$.

The Poisson point process $N$ describes the asymptotic behaviour of the sample clouds in the direction $\boldsymbol{q}$. The normalized sample clouds have the form

$$
N_{n}=\left\{\boldsymbol{W}_{n 1}, \ldots, \boldsymbol{W}_{n n}\right\}, \quad \boldsymbol{W}_{n i}=\frac{\boldsymbol{X}_{i}-b_{n} \boldsymbol{q}}{a_{n}}, \quad i=1, \ldots, n,
$$

where $\boldsymbol{X}_{1}, \ldots, \boldsymbol{X}_{n}$ are independent observations from $f$ and the coefficients $a_{n}>0$ and $b_{n}$ depend on the function $f_{0}$. Weak convergence $N_{n} \Rightarrow N$ holds on certain half-spaces. It holds 
on all half-spaces $\{\xi \geq c\}, c \in \mathbb{R}$, for which $\xi \boldsymbol{q}$ is positive and for which the intersection of the half-space $\{\xi \geq-1\}$ and the cone $Q$ is bounded. These linear functionals $\xi$ form an open convex cone $\Gamma$ in the dual space.

The conditions on the set $D$ and on the density generating function $f_{0}$ are minimal. The condition that the density $f$ is homothetic is severe. It may be relaxed slightly. The results remain valid for any density which is asymptotic to $f$ for $\|\boldsymbol{x}\| \rightarrow \infty$, and $f(\boldsymbol{x})>0$. The weaker assumption that the level sets of the density asymptotically have the shape $D$ will ensure that the sample clouds properly scaled converge onto the closure of the set $D$, but this condition is too weak to handle the asymptotic behaviour of the sample clouds under the normalization $\boldsymbol{w}=\left(z-b_{n} \boldsymbol{q}\right) / a_{n}$ above; see Example 5.1. The sensitivity of the limit to asymptotically negligible variations in the shape of the level sets in the case of asymptotic dependence is related to the scale function associated with the density generator.

If we assume asymptotic dependence with an exponent measure which does not charge points at $-\infty$ and which has a continuous density on $\mathbb{R}^{d}$, and if we assume that the associated normalizations are scalar, of the form $\boldsymbol{u}=\left(\boldsymbol{x}-t_{n} \boldsymbol{q}\right) / a_{n}$ for $a_{n}>0$ and $t_{n} \rightarrow \infty$, as in (3.4), then it is not unreasonable to assume a continuous density $\tilde{f}$ for the vector, and convergence of the normalized densities as in (3.4). In order to prevent sample points from wandering off to infinity after normalization, we need $\mathcal{L}^{1}$-convergence on the $d$ coordinate half-spaces $\left\{x_{i} \geq 0\right\}$. This means that the density $\tilde{f}$ has to be well behaved in any sequence of points $\boldsymbol{x}_{n}$ for which one of the coordinates tends to $+\infty$, even if the other coordinates all diverge to $-\infty$. A simple way to achieve this is by imposing the condition

$$
\left\{\tilde{f}>\mathrm{e}^{-t}\right\} \subset\left\{f>\mathrm{e}^{-t}\right\}, \quad t \geq 0
$$

on the level sets, where $f$ has homothetic level sets and its density generator $f_{0}$ is a von Mises function to ensure that the size of its level sets does not increase too fast. These conditions ensure that our results for homothetic densities carry over to the density $\tilde{f}$; compare Theorem 3.1 and Proposition 5.2.

Although geometric arguments often provide a better insight into complex analytic expressions, the geometry is not always simple and, as the counterexamples in Appendix A demonstrate, our intuition for star-shaped sets is not well developed, even in dimension $d=2$. (Example A.4 exhibits a fern-like set $D$ whose convex hull is the triangle in Figure 1(a). The set $D$ is sharp, but the components of the vector $\boldsymbol{U}$ uniformly distributed on $D$ are asymptotically independent, as are the components of the vector $\boldsymbol{X}$ with density $c_{0} \mathrm{e}^{-n_{D}}$.) There are many open problems here. Does asymptotic independence of the components of $\boldsymbol{U}$ uniformly distributed on $D$ imply asymptotic independence of the vector $\boldsymbol{X}$ with density $c \mathrm{e}^{-n_{D}}$ ? Does asymptotic independence of the components of the vector $\boldsymbol{X}$ with this density imply asymptotic independence for all densities $f_{0}\left(n_{D}\right)$ with a von Mises function $f_{0}$ ? The theory developed above enriches our understanding of multivariate extremes. For applications, the most pressing questions seem to be of a more statistical nature. How does one decide from a given sample whether the underlying distribution, assuming a homothetic density, has a shape $D$ with a sharp vertex? How does one estimate the vertex $\boldsymbol{q}$ and the associated cone $C$ ? For samples from a homothetic light-tailed density $f=f_{0}\left(n_{D}\right)$, the scaled sample clouds converge onto the closure of $D$. Given a finite sample sequence, how does one test whether the underlying density is homothetic? 


\section{Appendix A}

\section{A.1. Counterexamples}

Example A.1. Let $\boldsymbol{q}$ be the vertical unit vector in $\mathbb{R}^{3}$. Let $h$ have level sets

$$
\left\{h>\mathrm{e}^{-t}\right\}=(-\infty, 0)^{3}+t \boldsymbol{e}_{3}=C+t \boldsymbol{q}, \quad t \in \mathbb{R} .
$$

Then $\{h>0\}=(-\infty, 0)^{2} \times \mathbb{R}=C+\mathbb{R} \boldsymbol{q}$ and $h$ is discontinuous in all boundary points.

Example A.2. Let $E$ be a bounded open set in the plane given by the union of the unit ball $B$ and the open triangles $T$ and $-T$, where $T$ has its top in the origin and base vertices $( \pm 1,-1)$; see Figure 4(a). The set $E$ is star shaped and contains the origin, but its boundary is not continuous. No proper translation of $E$ is star shaped. Let $C$ denote the open cone in $\mathbb{R}^{3}$ which intersects the plane $\{z=-1\}$ in $E \times\{-1\}$. There exists a set $D \in D_{3}$ having $\boldsymbol{e}_{3}$ as a boundary point, such that $D-\boldsymbol{e}_{3}$ generates $C$.

The set $D$ is a subset of $D_{1}=\left(C+\boldsymbol{e}_{3}\right) \cap\{z>-1\}$, but its base is the open disk $R$ of radius 2 in $\{z=-1\}$ rather than $2 E \times\{-1\}$. The boundary of $D_{1}$ contains the open triangle $T_{1}$, with top $\boldsymbol{e}_{3}$ and base vertices $( \pm 2,-2,-1)$, and its reflection $\tilde{T}_{1}$, with top $\boldsymbol{e}_{3}$ and base vertices $( \pm 2,2,-1)$. Define $S \subset T_{1}$ to be the intersection of two open disks centred in $( \pm s,-1,0)$ whose boundary circles intersect in $\boldsymbol{e}_{3}$ and $(0,-2,-1)$, and are tangent to the sides of the triangle $T_{1}$ in $\boldsymbol{e}_{3}$. The set $\tilde{S} \subset \tilde{T}_{1}$ is defined similarly. We describe $D$ in terms of its intersections $D(a)$ with the planes $H(a)=\{y=a x\}$. For $|a| \leq 1$, the intersection is an open triangle. The top is $\boldsymbol{e}_{3}$; the base is the intersection of the disk $R$ and $H(a)$. For $|a|>1$, the intersection $D(a)$ is the open convex subset of $H(a)$ generated by the intervals $R \cap H(a), S \cap H(a)$, and $\tilde{S} \cap H(a)$. Now observe that the set $D(a)$ varies continuously with $a \in[-\infty, \infty]$ and that $D(a)-\boldsymbol{e}_{3}$ generates the cone $C \cap H(a)$ for any $a$.

Example A.3. Let $C$ be the open cone in $\mathbb{R}^{3}$ generated by the union $E$ of a disk and two triangles with a common vertex at the origin in the horizontal plane $\{y=-1\}$, as in Example A.2.

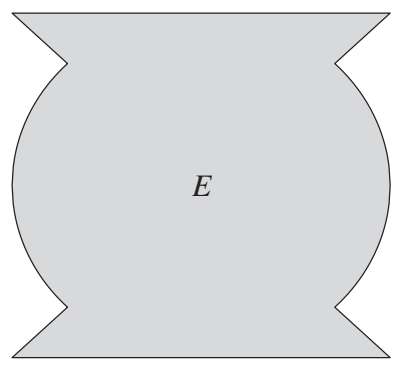

(a)

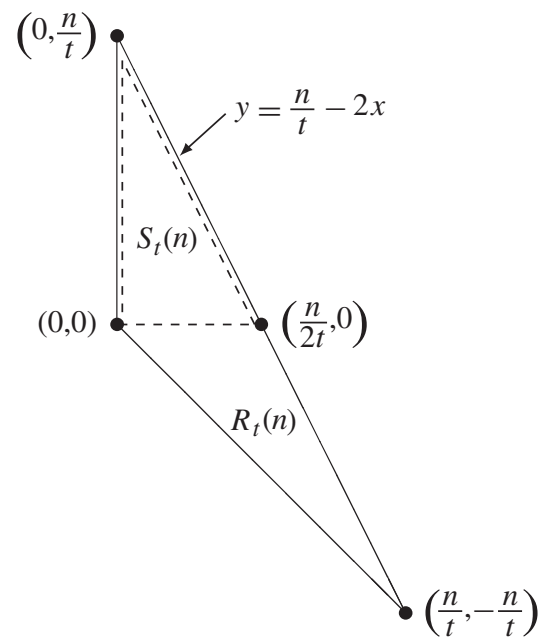

(b)

Figure 4: (a) The set $E$ used in Examples A.2 and A.3. (b) The triangles $S_{t}(n)\left(\right.$ dashed lines) and $R_{t}(n)$ (solid lines); $S_{t}(n) \subset R_{t}(n)$ (see Example A.4). 
Suppose that the function $h$ with level sets $\left\{h>\mathrm{e}^{-t}\right\}=C+t e_{3}$ is continuous. Then the set $\{h=1\}$ restricted to $\{y=-1\}$ is $\partial E$; the set $\left\{h=\mathrm{e}^{-\varepsilon}\right\}$ is the boundary of the same set enlarged by the factor $1+\varepsilon$. For small $\varepsilon>0$, there are points which lie on both boundaries. At these points the function values are equal, so $1=1+\varepsilon$, a contradiction.

Example A.4. For the open triangle $T$ with vertices $(1,1),(-1,0)$, and $(0,-1)$ (see Figure $4(\mathrm{a}))$, the components of the vector $(X, Y)$ with density $c \mathrm{e}^{-n_{T}}$ are asymptotically dependent. There is a limit vector $(U, V)$ for the coordinatewise maxima. The components have a Gumbel distribution, but they are dependent. The exponent measure has a continuous density $h$ on $\mathbb{R}^{2}$ with level sets which are translates over $(t, t)$ of a cone in the negative quadrant. The density $h$ has the form $h(u, v)=\frac{2}{3}\left(\mathrm{e}^{u-2 v} \wedge \mathrm{e}^{v-2 u}\right)$ with exponential margins $\mathrm{e}^{-u}$ and $\mathrm{e}^{-v}$. One might expect the continuous densities $c \mathrm{e}^{-n_{D}}$ to be asymptotically dependent for any open star-shaped set $D$ whose convex hull is the triangle $T$.

Asymptotic independence may occur even if the shape set $D$ has a sharp vertex in the point $\sup D$.

Below we construct a star-shaped open set $D$ which lies in the open triangle $T$ above. The set $D$ contains the origin, it is symmetric around the diagonal, it has a continuous boundary, and $T$ is its convex hull. Yet the random vector with density $c \mathrm{e}^{-n_{D}}$ has asymptotically independent components.

We shall first construct a simpler set $D_{0}$ which satisfies the conditions above, except for the continuity of the boundary. The set $D_{0}$ is the union of $T_{0}=T \backslash\left[\frac{1}{2}, \infty\right)^{2}$ with a sequence of open sets which are the intersection of the triangle $T$ with disjoint thin open cones $C_{n}$ converging to the positive diagonal from below, and a similar sequence of cones converging from above. The set $D_{0}$ looks like an arrangement of grasses thinning out towards the top in the vase $T_{0}$. We shall treat the part below the diagonal.

Choose a decreasing sequence of disjoint intervals $J_{n}=\left(a_{n}, b_{n}\right)$ in $(0,1)$ with

$$
a_{0}=1>b_{1}>a_{1}>b_{2}>\cdots, \quad a_{n+1} \sim a_{n} \rightarrow 0, \quad b_{n}-a_{n} \ll a_{n}-b_{n+1} .
$$

Define $C_{n}$ to be the open cone spanned by the interval $\left(1+a_{n}, 1+b_{n}\right)$ on the horizontal line $y=1$. With the union $J$ of the intervals $J_{n}$ we associate a DF $G$ on $(0, \infty)$ with density $\mathbf{1}_{J}$ on $(0,1)$ and $G(0)=0$. Then $G(1)=|J|$ is the total length of the intervals $J_{n}$. We choose the intervals $J_{n}$ such that

$$
G(x) \sim F(x):=\mathrm{e}^{-(\log x)^{2}} \text { as } x \rightarrow 0 .
$$

The function $F$ is a DF on $(0,1)$, continuous, and strictly increasing. It goes to 0 faster than any power for $x \rightarrow 0$. The set $J$ thus has a very low density in the neighbourhood of 0 . This also holds for the density of the set $D_{0}$ in the neighbourhood of the vertex $(1,1)$ since $D_{0}$ is the union of the sets $C_{n} \cap T$ on $T \cap\left\{\frac{1}{2} \leq y<x\right\}$. It explains why the density $c_{0} \mathrm{e}^{-n_{D_{0}}}$ behaves asymptotically as if $D_{0}$ were blunt.

Here are the details. Expand the set $D_{0}$ by the factor $t+n$ with large $t$, let $p_{t}(n)$ denote the area of $(t+n) D_{0}$ in the shifted quadrant $(t, \infty)^{2}$ below the diagonal, and let $q_{t}(n)$ denote the area in $\{x>t\}$ below the diagonal. Obviously,

$$
p_{t}(n) \leq q_{t}(n), \quad p_{t}(n) \leq p_{t}(n+1), \quad q_{t}(n) \leq q_{t}(n+1), \quad n=1,2, \ldots, t \geq 0 .
$$

A closer look at the density, see below, will show that $p_{t}(n) \ll q_{t}(n)$ for $t \rightarrow \infty$, provided that $n \leq \sqrt{t}$. (Thus, Sibuya's condition holds for the uniform distribution on $D_{0}$.) Introduce the 
sums

$$
P(t)=\sum_{n=1}^{\infty} \frac{p_{t}(n)}{\mathrm{e}^{n}}, \quad Q(t)=\sum_{n=1}^{\infty} \frac{q_{t}(n)}{\mathrm{e}^{n}}, \quad t>1 .
$$

We shall prove that $P(t) / Q(t)$ vanishes for $t \rightarrow \infty$. This is Sibuya's condition for asymptotic independence of the density $c_{0} \mathrm{e}^{-n_{D_{0}}}$. Indeed, nonnegative functions may be approximated by step functions, writing $g / \mathrm{e} \leq(1-1 / \mathrm{e}) \sum \mathrm{e}^{-n} \mathbf{1}_{\left\{g>\mathrm{e}^{-n}\right\}} \leq g$. On the complement of $(-\infty, t]^{2}$,

$$
\frac{\mathrm{e}^{-n_{D_{0}}}}{\mathrm{e}} \leq\left(1-\frac{1}{\mathrm{e}}\right) \mathrm{e}^{-t} \sum_{n=1}^{\infty} \mathrm{e}^{-n} \mathbf{1}_{(t+n) D_{0}} \leq \mathrm{e}^{-n_{D_{0}}}
$$

The integral of the step function (without the additional factor $\left.(1-1 / \mathrm{e}) / \mathrm{e}^{t}\right)$ over the shifted quadrant $(t, \infty)^{2}$ is $2 P(t)$; the integral over the half-plane $\{x>t\}$ is $Q(t)+P(t)$. The condition $P(t) / Q(t) \rightarrow 0$ implies that the quotient of the integrals of $\mathrm{e}^{-n_{D_{0}}}$ over these two sets also vanishes, which is Sibuya's condition.

In order to handle these infinite series, we show that

$$
\frac{p_{t}(n)}{q_{t}(n)} \rightarrow 0 \quad \text { as } t \rightarrow \infty \text {, uniformly in } n \text { for } 1 \leq n \leq \sqrt{t}
$$

For $n \geq \sqrt{t}$, the terms of the series for $P(t)$ may be bounded by $n^{2} / 4 \mathrm{e}^{n}$, where $n^{2} / 4$ is just the area of the triangle $(t+n) T \cap\{t \leq y<x\}$ with vertices $(t, t),(t+n, t+n)$, and $(t+n / 2, t)$. Hence, the tail, $n \geq \sqrt{t}$, of the series for $P(t)$ is bounded by $t \mathrm{e}^{-\sqrt{t}}$ for $t>0$. We shall show that $t \mathrm{e}^{-\sqrt{t}} / q_{t}(1)$ vanishes for $t \rightarrow \infty$. The bound is valid for any open set $D \subset T$ containing $D_{0}$.

To see why $p_{t}(n) / q_{t}(n)$ vanishes for $1 \leq n \leq \sqrt{t}$, we introduce new coordinates. Make the diagonal the new vertical axis, but retain the horizontal axis. The point $(t, t)$ has now moved to $(0, t)$. We make this point our new origin by a vertical translation of the coordinates. Now $p_{t}(n)$ is the weight of the triangle $S_{t}(n)$ with vertices $(0,0),(0, n)$, and $(n / 2,0)$ with respect to the measure $\mu$ with density $\mathbf{1}_{(t+n) D_{0}}$, and $q_{t}(n)$ is the weight of the triangle $R_{t}(n)$ with vertices $(0,0),(0, n)$, and $(n,-n)$. Define $\mu^{*}$ as the measure with density $\mathbf{1}_{t J \times \mathbb{R}}$. In the new coordinates the cone $C_{m}$ has top $(0,-t)$ and intersects the horizontal axis in the interval $t J_{m}$. It is replaced by the strip $t J_{m} \times \mathbb{R}$ to obtain the density of $\mu^{*}$. For $n=o(t)$, the masses $\mu\left(S_{t}(n)\right)$ and $\mu^{*}\left(S_{t}(n)\right)$ are asymptotic, and, hence, $p_{t}(n)=\mu\left(S_{t}(n)\right) \leq 2 p_{t}^{*}(n):=\mu^{*}\left(S_{t}(n)\right)$ for $t \geq t_{0}$ and $1 \leq n \leq \sqrt{t}$. Similarly, when $t J_{m} \subset(0, n)$, the area of $t J_{m} \times \mathbb{R}$ in $R_{t}(n)$ is asymptotic to the area in $R_{t}(n)$ due to the cone $C_{m}$, and, hence, $q_{t}(n) \geq q_{t}^{*}(n) / 2$ for $t \geq t_{1}$.

It is clear why (A.1) holds. The density of the set $t J \times \mathbb{R}$ increases as one moves away from the vertical axis. The triangle $R_{t}(n)$ extends twice as far to the right as $S_{t}(n)$. Both triangles have the same base on the vertical axis. The fraction $F(n / 2 t) / F(n / t)$ gives an indication of the size of $p_{t}(n) / q_{t}(n)$, as we show next. Set $F_{1}(x)=\int_{0}^{x} F(t) \mathrm{d} t$. Then $F_{1}(x) \sim x F(x) / 2 \log (1 / x)$ for $x \rightarrow 0$. By partial integration,

$$
\frac{p_{t}^{*}(n)}{t^{2}}=\int_{0}^{n / 2 t}\left(\frac{n}{t}-2 x\right) \mathrm{d} G(x)=2 \int_{0}^{n / 2 t} G(x) \mathrm{d} x \sim 2 \int_{0}^{n / 2 t} F(x) \mathrm{d} x=2 F_{1}\left(\frac{n}{2 t}\right),
$$

and, similarly, $q_{t}^{*}(n) / t^{2} \sim F_{1}(n / t)$ for $n / t \rightarrow 0$. Hence,

$$
\frac{p_{t}^{*}(n)}{q_{t}^{*}(n)} \sim \frac{2 F_{1}(n / 2 t)}{F_{1}(n / t)} \sim \frac{F(n / 2 t)}{F(n / t)}=c\left(\frac{n}{t}\right)^{\log 4} \text { as } \frac{n}{t} \rightarrow 0, c=F\left(\frac{1}{2}\right),
$$


and (A.1) holds. The relation $q_{t}^{*}(n) \sim t^{2} F_{1}(n / t)$ above for $n=1$ gives $q_{t}(1) \ll t F(1 / t) \gg$ $t \mathrm{e}^{-\sqrt{t}}$ for $t \rightarrow \infty$. This shows that the tail, $n \geq \sqrt{t}$, of the series for $P(t)$ does not contribute to the fraction $P(t) / Q(t)$. Asymptotic independence for the density $c_{0} \mathrm{e}^{-n_{D_{0}}}$ follows from $P(t) / Q(t) \rightarrow 0$.

We still have to replace $D_{0}$ by a star-shaped set $D$ with a continuous boundary without changing the asymptotics of (A.1). This is done in two steps: $D_{0} \subset D_{1} \subset D$, increasing $p_{t}(n)$ and $q_{t}(n)$ each step. It suffices to check that $p_{t}(n)$ for $1 \leq n \leq \sqrt{t}$ and $t>t_{2}$ increases by no more than a factor of 2 in either step.

First, replace the cones $C_{n}$ in the construction of $D_{0}$ by strips $L_{n}$ which intersect the horizontal line $y=1$ in the interval $\left(1+a_{n}, 1+b_{n}\right)$ and the horizontal axis in the interval $\left(-d_{n} / 2, d_{n} / 2\right)$, where $d_{n}=b_{n}-a_{n}$. This yields an open set $D_{1} \subset T$ whose boundary is continuous outside the vertex $(1,1)$. Second, add an open spike $S \subset T$, symmetric around the diagonal, whose upper boundary is given by the equation $y=x+F((1-x) / 3)$, $\frac{1}{2} \leq x<1$. The set $D=D_{1} \cup S$ has a continuous boundary. In the new coordinates the spike ends at $(0, n)$ and the right boundary is $x=(t+n) F((n-y) / 3(t+n))$. Now use $\int_{0}^{\varepsilon} F(v / 3) \mathrm{d} v=3 F_{1}(\varepsilon / 3)$ with $\varepsilon=n /(t+n)$ and scale. The quantities $p_{t}(n)$ increase by less than $(t+n)^{2} 3 F_{1}(\varepsilon / 3) \ll 2 t^{2} F_{1}(n / 2 t)$. Hence, (A.1) holds for the quantities $p_{t}(n)$ and $q_{t}(n)$ associated with the set $D$, and Sibuya's condition applied to the continuous homothetic density $c \mathrm{e}^{-n_{D}}$ yields asymptotic independence.

\section{A.2. Supplementary results}

A.2.1. Asymptotic independence. To keep the paper self-contained, this section gives two criteria for asymptotic independence. For a detailed discussion of the notion of asymptotic independence and proofs, the reader is referred to [2].

Consider a random vector $\left(X_{1}, X_{2}\right)$ with the joint DF $F$ and marginal DFs $F_{1}$ and $F_{2}$. Define a function $P$ by $P\left(F_{1}\left(x_{1}\right), F_{2}\left(x_{2}\right)\right)=\operatorname{Pr}\left\{X_{1}>x_{1}, X_{2}>x_{2}\right\}$. Sibuya's condition (see [4, Theorem 2]) says that the components $X_{1}$ and $X_{2}$ are asymptotically independent if the function $P$ satisfies the following limit relation:

$$
P(1-s, 1-s)=o(s) \quad \text { as } s \rightarrow 0, s>0 .
$$

The following criterion for asymptotic independence is expressed in terms of a continuous curve, $\boldsymbol{x}(t)=\left(x_{1}(t), x_{2}(t)\right), t \geq 0$, for which $F_{1}\left(x_{1}(t)\right)$ and $F_{2}\left(x_{2}(t)\right)$ tend to 1 as $t \rightarrow \infty$. See Proposition 2.3 of [2].

Proposition A.1. Let $\left(X_{1}, X_{2}\right)$ have $D F F$ with continuous marginals $F_{1}$ and $F_{2}$. The components $X_{1}$ and $X_{2}$ are asymptotically independent if and only if, for any $\varepsilon>0$, there exists a continuous curve $\boldsymbol{x}(t), t \geq 0$, such that $p_{i}(t)=\operatorname{Pr}\left\{X_{i}>x_{i}(t)\right\}$ is positive and vanishes for $t \rightarrow \infty$ and $i=1,2$, and such that

$$
\frac{\operatorname{Pr}\left\{X_{1}>x_{1}(t), X_{2}>x_{2}(t)\right\}}{p_{i}(t)}<\varepsilon, \quad t>t_{0}, i=1,2 .
$$

\section{A.2.2. Miscellaneous.}

Proposition A.2. Suppose that $D \in \mathcal{D}_{d}$ and the half-space $H=\{\xi \geq c\}$ is disjoint from $D$ and contains the boundary point $\boldsymbol{q}$ of $D$. Let $D$ be asymptotic to the open cone $C$ in $\boldsymbol{q}$. Then $C$ lies in the open half-space $\{\xi<0\}$ and $C_{1}=C+\boldsymbol{q}$ is star shaped.

Proof. Suppose that $\boldsymbol{q} \neq \mathbf{0}$. (Otherwise, $C=D_{\infty}$ and the cone $C$ is convex.) Suppose that $z \in C_{1}$. The set $D$ contains all points on the interval $(\boldsymbol{q}, z)$ sufficiently close to $\boldsymbol{q}$ by 
Definition 2.3. Let it contain the interval $(\boldsymbol{p}, \boldsymbol{q})$. The set $D$ is star shaped. So it contains the triangle with vertices $\boldsymbol{p}, \boldsymbol{q}$, and $\mathbf{0}$, apart perhaps from the segment $[\mathbf{0}, \boldsymbol{q}]$ (if $\boldsymbol{q}$ lies on the boundary of the cone $D_{\infty}$ ). By Definition 2.3, $C$ contains this set, translated over $-\boldsymbol{q}$, and, hence, the cone generated by the translated set. The shifted cone contains the interval $(\mathbf{0}, z]$.

Lemma A.1. Suppose that the $g_{n}$ are measurable functions on the half-space $H \subset \mathbb{R}^{d}$ which satisfy $0 \leq g_{n} \leq M_{0}$ for $n \geq n_{0}$ and some constant $M_{0}$. Let $g_{n} \rightarrow g$ a.e. on $H$. If, for any $\varepsilon>0$, there exists a compact set $E$ such that $\int_{H \backslash E} g_{n}(\boldsymbol{x}) \mathrm{d} \boldsymbol{x}<\varepsilon$ for $n \geq n_{1}$ then $g_{n} \rightarrow g$ in $\mathcal{L}^{1}$ on $H$.

\section{References}

[1] Balkema, A. A. And Embrechts, P. (2007). High Risk Scenarios and Extremes. European Mathematical Society, Zürich.

[2] Balkema, A. A. And Nolde, N. (2010). Asymptotic independence for unimodal densities. Adv. Appl. Prob. 42, 411-432.

[3] Resnick, S. I. (1987). Extreme Values, Regular Variation, and Point Processes. Springer, New York.

[4] Sibuya, M. (1960). Bivariate extreme statistics, I. Ann. Inst. Statist. Math. 11, 195-210. 